\title{
42. CLOSE BINARY STARS (ETOILES DOUBLES SERREES)
}

PRESIDENT: G. Larsson-Leander

VICE-PRESIDENT: B. Warner

ORGANIZING COMMITTEE: A.H. Batten, A.M. Cherepashchuk, M.G. Fracastoro, K. Gyldenkerne, T.J. Herczeg, M. Kitamura, Y. Kondo, M. Plavec, S.D. Sinvhal, J. Smak, E.P.J. van den Heuvel, J.A.J. Whelan

\section{Introduction}

Studies of close binary stars were persued more vigorously than ever: about 3000 research papers and notes pertaining to the field were published during the triennium 1976 - 1978. Many major advances and spectacular discoveries were made, mostly due to increased observational efficiency and precision, especially in the $X-r a y$, radio, and ultraviolet domains. The high activity is also shown by the number of conferences devoted to close-binary problems.

The proceedings of our 1975 Cambridge Symposium, IAU No. 73, appeared in print in 1976: Structure and Evolution of Close Binary Systems, eds. P. EgBleton, S. Mitton, and $\mathrm{J}$. Whelan; Reidel Publ. Co. Likewise, IAU Symposium No. 70, dedicated to the memory of P.W. Merrill and D.B. McLaughlin, was printed by Reidel in 1976 (Be cond She IZ Stars, ed. A. Slettebak). At the Grenoble Meeting decision was taken to co-sponsor a symposium on "Mass-Loss and Evolution of 0-type Stars". It was held, as IAU Symposium No. 83, at Qualicum Beach, Vancouver Island (Canada), in June 1978; many papers and much of the discussion dealt with close binaries. As will be known to all readers, the Commission is sponsoring IAU Symposium No. 88, "Close Binary Stars: Observation and Interpretation", to be held in Toronto, August 1979, with M. Plavec as Chairman of the Scientific Organizing Committee. Further, the Commission offered co-sponsorship for a symposium on "Fundamental Problems in the Theory of Stellar Evolution", planned for Kyoto, July 1980. IAU Colloquia of special interest were: No. 29, Multiple Periodic Variable Stars, ed. W.S. Fitch, published 1976 in two volumes by the Hungarian Academy of Sciences (Vol. I, with the invited papers, was also issued by Reidel); No. 33, "Observational Parameters and Dynamical Evolution of Multiple Stars", published 1977 as Vol. 3 of Rev. Mexicana Astron. Astrophys.; No. 42, "The Interaction of Variable Stars with their Environment" appeared in book form 1977 (eds. R. Kippenhahn, J. Rahe, and W. Strohmeier, Veröff. Remeis-Stermw. Bamberg, No. 12l) just a few months after the colloquium; No. 46, "Changing Trends in Variable Star Research", held at Hamilton, New Zealand, November 1978. The conference on "X-ray Binaries" at Goddard Space Flight Center in October 1975 was edited by E. Boldt and Y. Kondo and published early 1976 as NASA SP-389. Reidel printed 1977 Novae and Related Stars (ed. M. Friedjung), the proceedings of the September 1976 Paris conference. The 1974 Moscow conference on evolutionary problems was followed up by a second conference in Warsaw, June 1977 (Nonstationary Evolution of Close Binaries, ed. A.N. Zytkow, Polish Scientific Publ.,1978).

Two important monographs were published, viz., Z. Kopal, Dynamics of Close Binary Systems, Reidel Publ. Co., 1978, and J. Sahade and F.B. Wood, Interacting Binary Stars, Pergamon Press, 1978. Of utmost importance for close-binary workers is the appearance 1978 of Seventh Catalogue of the Orbital Elements of Spectroscopic Binary Systems, by A.H. Batten, J.M. Fletcher and F.J. Mann (Publ. Dominion Astrophys. Obs. 15, No. 5). Also, we should mention that a new edition of the Finding List for obsemers of Eclipsing Variables will soon be published by F.B. Wood, R.H. Koch, J.P. Oliver, and D.R. Florkowski. The Bibliography and Program Notes on Close Binaries continued to be issued from Lund Observatory, with G. Larsson-Leander as editor. Nos. 27 - 31 appeared during the triennium; regional contributors were B. Cester (Italy), M. de Groot and R.F. Sisteró (Southern Hemisphere), J. Grygar (Central and Eastern Europe), D.S. Hall and 
G.W. Henry (USA, except the West Coast), M. Kitamura (Japan); H. Mauder (Germany and IAU Circ.), C.D. Scarfe (Canada, US West Coast, Mexico), A. Shulberg (USSR), S.D. Sinvhal and C.D. Kandpal (India and Indonesia), F. van't Veer (Western Europe).

The present Report is based on published literature and information from Commission members. Members of the Organizing Committee kindly consented to write specific Sections, and for reviewing radio observations I got the expert help of Dr. D.M. Gibson. The names of these contributors are indicated at proper places. In addition, on my request $\mathrm{Dr}$. A.M. Cherepashchuk wrote an extensive summary of the work done in the USSR, excerpts of which were communicated to the contributors. However, to keep the Report at the prescribed length, make the presentation as homogeneous as possible, and take into account the most recent literature, the contributions were more or less heavily edited. Much material was especially added to Tables 1 - 4, in an attempt to make them as complete as possible. Lack of space made it necessary to omit names of authors in the tables. As it was realized that many details on work in progress could not be included in the Report, much of this information was given in No. 31 of the Bibliography. I am deeply thankful to all contributing authors and Commission members who have helped me so generously.

Key to the references:

$A A=$ Acta Astron.

$A A p=$ Astron. Astrophys.

AAp Sup = Astron. Astrophys., Suppl. Ser. AASin = Acta Astron. Sinica

Izv Krym = Izv. Krymskoj Astrofiz. Obs. $J A A V S O=J$. American Assoc. Variable

Af $=$ Astrofizika

Af Issl = Astrofiz. Issled. Izv. Spets. Astrofiz. Obs.

$A J=$ Astron. $J$.

AmAf = Astrometr. Astrofiz., Kiev

$A N=$ Astron. Nachr.

Ann Rev $A A p=$ Annu. Rev. Astron. Astrophys.

Ann Tokyo = Ann. Tokyo Astron. Obs., Second Ser.

ApJ $=$ Astrophys. J.

ApJ Sup = Astrophys. J., Supp Z. Ser.

$A p L=$ Astrophys. Lett.

ApSpSc = Astrophys. Space Sci.

$A T s=$ Astron. Tsirk.

$A Z h=$ Astron. Zh. Akad. Nauk SSSR

$B A A S=$ Buzl. American Astron. Soc.

$B A C=$ Bulz. Astron. Inst. Czechosiovakia

Bamberg Ver = Veröff. Remeis-Stemw. Bamberg

$B A S I=$ Bulz. Astron. Soc. India

Byulz Abastuman = ByulZ. Abastuman. Astrofiz. Obs.

Com Ankara = Commun. Astron. Dep. Univ. Ankara

ComAp = Comments Astrophys.

Contr CZuj-Napoca = Contrib. Astron. Obs. univ. "Babes-Bolyai", Cluj-Napoca IAUC $=$ IAU Circ.

IBVS = Inf. Bulz. Variable stars

Izv $A N$ AzSSR = Izv. Akad. Nauk Azerb. SSR, Ser. fiz.-tekn. i mat. n.

Izv Ehngelg = Izv. Astron. Ehngel'gardt. Obs., Kazan Star Obs.

JRASC $=J . R$. Astron. Soc. Canada

Kodaikanal BulZ = Kodaikanal Obs. Bulz.

Liège Colz = Colzoque Int. Astrophys. Liège

MittAG = Mitt. Astron. Ges.

$M N=$ Mon. Not. R. Astron. Soc.

MRAS $=$ Mem. R. Astron. Soc.

$M S A I=$ Mem. Soc. Astron. Italiana

$O b s=$ Observatory

PASP $=$ Publ. Astron. Soc. Pacific

Perzv = Perem. Zvezdy, Byuzz.

Perzv Pril = Perem. Zvezdy, Prizozhenie

Pis $A 2 h=$ Pis'ma $v$ Astron. $2 h$.

Publ DAO = Publ. Dominion Astrophys. Obs.

Publ Tartu = Publ. Tartu Astrofiz. Obs.

QJRAS $=$ Q. J. R. Astron. Soc.

Rep Ege = Sci. Rep. Fac. Sci. Ege Univ., Izmir

Rep Lund $=$ Rep. Obs. Lund

Soob ZeZenchuk = Soobshch. Spets. Astrofiz. Obs.

Stud Babes-Bolyai = Stud. Univ. BabesBolyai, Ser. Math.-phys.

Tokyo Bull = Tokyo Astron Bull., Second Ser.

Trudy Alma-Ata = Trudy Astrofiz. Inst. $A$ Ima-Ata

Trudy Kazan = Trudy Kazan. Gorod. Astron. Obs.

Ts Lvov = Tsirk. Astron. Obs., L'vov.

Ts Shemak = Tsirk. Shemakhinsk. Astrofiz. Obs.

Vilianova Contr = Vilzanova univ. Obs. contrib.

Vistas = Vistas Astron. 
A wide range of techniques are currently used for the study of close binary stars. The more conventional photometric and spectrographic observations, polarimetry, $X$-ray and radio observations are discussed in Section 4. Extraterrestrial observations, including those made with the IUE, are summarized in Section 10. In addition we mention here the uItraviolet satellite observations of $B$ Lyr (Kondo et al., ApSpSc 41,121), V1500 Cyg (Jenkins et al., ApJ 212,198; Wu and Kester, AAp 58,331), W UMa (Rucinski, $A A \underline{26}, 227$ ), and V Pup (York et al., ApJ 210,143).

High-resolution spectroscopy of HR Del (Gallagher and Anderson, ApJ 203,625) used an Echelle system, in V1500 Cyg photoelectric scans gave good results for interstellar lines (Tomkin et al., AAp 48,319). Infrared spectroscopy $(2-3 \mu$ ) detected coronal lines in V1500 Cyg (Grasdalen and Joyce, Nature 259,187); 1 - $20 \mu$ spectra of this nova were obtained by Ennis et al. (ApJ 214,478). An application of the surface-brightness technique to V1500 Cyg (Barnes, $\overline{M N}$ 177,53P) provided a new way of estimating distances to novae.

Rapid photometry was widely used for studies of dwarf and other novae (Nevo and Sadeh, MN 177,167, 182,595; Robinson and Nather, PASP 89,572; Szkody, ApJ 207,190; Warner and Brickhill, $M N$ 182, 777 ), quasi-periodic variations were found in VW Hyi (Haefner et al., AAp 61,L37), RU Peg (Patterson et al., ApJ 214,144), and WZ Sge (Robinson et al., ApJ 219,168). Rapid flickering was detected in AM Her (Bailey et al., MN 180,35P) and in the symbiotic variable $\mathrm{CH}$ Cyg at outburst (Slovak and Africano, $M N$ 185,591). Optical pulsations were detected in HZ Her (Middleditch and Nelson, ApJ 208, 567) and from 4U 1626-67 (Ilovaisky et al., AAp 70,L19). Optical bursts were reported from MXB 1735-44 (MCClintock et al., Nature 274,567). Rapid variations in line profiles were seen in V1500 Cyg (Campbell, ApJ 207, L41), in EZ CMa (Vojkhanskaya et al., Af 12,180), and in B Lyr (Sanyal, ApJ 210, 853). Pulse-resolved spectrophotometry was obtained for DQ Her by Margon et al. (ApJ 208,L35), and possible pulsations in the spectral lines were reported (Margon et al., PASP $\underline{89}, 300$ ).

We note the increasing importance of speckle interferometry (Blazit et al., ApJ 214,L79; McAlister, ApJ 215,159, 225,932; Morgan et al., MN 183,701) for spectroscopic binaries. Detailed studies by McAlister include $n$ Ori (PASP 88,957), 12 Per (ApJ $223,526)$, and 51 Tau (ApJ 212,459). Lunar occultations continue to furnish information on new and old systems (e.g., Africano et al., AJ 82,631, 83,1100). Long-focus astrometry by van de Kamp yielded parallaxes and orbital data for $\varepsilon$ Aur $(A J$ 83,975) and VV Cep $(A J$ 82,750). New interferometric techniques (Currie et a2., BAAS 2,625) seem very promising.

\section{Methods of Analyzing Light Curves}

The methods of analyzing light changes in the frequency domain were further developed, primarily by Kopal and his collaborators in the long series of papers entitled "Fourier Analysis of the Light Curves of Eclipsing Variables" in ApSpSc (Vol. $34-57,19$ papers). Other contributions were given by Jurkevich et al. (ApSpSc $44,63)$, Rovithis-Iivaniou (ApSpSe 51,77, 22,271), and by several investigators dealing with specific systems.

As outlined by Kopal (ApSpSe 34,431), one of the most suitable methods of transforming from the time to the frequency domain is to consider light loss to be a function of even powers of the sine of the phase angle and to evaluate certain integrals $A_{2 m}$, called moments, from which the elements are determined. The relations between the $A_{2 m}$ and the geometrical elements, although simple for uniformly bright stars, were found complicated for limb-darkened stars. A simpler and more direct method to obtain these relations was indicated by Smith (ApSpSC 40,315). Kopal (ApSpSc 46,87) generalized the procedure, introducing the complete Fourier transform and considering 
also odd and non-linear moments. In Paper XI of the series, Kopal (ApSpSC 50,225) defined the light loss as the cross correlation of two apertures, representing the eclipsing and eclipsed disks. This new approach is shown to have considerable advantages compared to the geometrical one. The fractional light loss of arbitrarily limbdarkened stars, equal to the associated alpha-functions $\alpha_{2}^{Q}$, may be described as Hankel transforms of products of two Bessel functions, with orders depending on the physical characteristics of the components, while the geometry of the system enters only through their arguments. Kopal (APSpSc 51,439) gave explicit forms for the $\alpha_{i}^{0}$ for different types of eclipses together with the corresponding moments $A_{2 m}$, and alternative forms of the $\alpha_{i}^{0}$ were given by Demircan (ApSpSc 52,189). Kopal and Demircan (ApSpSe 55,241) described the practical procedures for deriving the geometrical elements for any type of eclipse, any proximity of components, and any limb-darkening. Demircan (ApSpSC 56,389) gave new general expressions for the moments $A_{2 m}$, allowing an automated method of analysis, and discussed (ApSpSc 56,453) which moments should be chosen for best determinacy. Photometric perturbations were considered by Edalati and Budding (ApSpSc 57,181) and by Kopal (ApSpSc 57,439). All the above papers assume circular orbits. The generalization to excentric orbits was given by Kopal and AlNaimiy in Paper XIX (ApSpSC 57,479). Several more papers of the series are in press. A book on the Fourier methods has already been completed by Kopal in manuscript form. It should be available as preprint to interested workers at the end of 1978, and will hopefully appear from the printer during 1979.

A computer program, based on Kopal's analytical method for solution in the time domain, was worked out by Söderhjelm (Rep Lund 10). Botsula (Izv Ehngelg 39,99) and the Peking Research Group (AASin 18,68) discussed the convergence of successive approximations in Kopal's method. Lanzano (ApSpSc 42,425, 45,419,483) discussed the $J$-integrals and derived recursion formulae useful for numerical computations.

Lavrov (PerZv Pril 2,349) published a program suited to the rectifiable RussellMerrill method, and coefficients for polynomials approximating the $\alpha(p, k)$ functions were tabulated by Lavrov and Sokolova (BAC 27, 301). Horak (BAC 26,257) presented a modification of his iterative minimization method. Ureche (Contr Cluj-Napoca, p. 13; Stud Babes-Bolyai, in press) discussed the influence of the quadratic and a possible sine term in the limb-darkening law, and he extended his studies of the ellipsoidellipsoid approximation to the case of elliptic orbits (BAC, in press). Budding reports that a 16-parameter curve-fitting program was put in operation in Manchester.

Binnendijk in a lucid paper (Vistas 2l,359) described a new method for computing light curves and deriving orbital parameters for contact binaries, assuming Roche potentials. A slightly more theoretical treatment was presented by Sapar (Publ Tartu 44,124). Eaton (BAAS 9,624$)$ wrote a program for application on RS CVn systems, assuming unevenly distributed spots on one component. Eclipses by an elliptical torus were treated by Wilson $(A J \underline{80}, 719)$.

Among the various existing programs for light-curve synthesis, especially those of D.B. Wood and Wilson and Devinney have been used extensively by many workers. For the benefit of users, Wood issued a number of WINK Status Reports, with improvements and amendments. In the Wilson-Devinney program, Wilson added an optional stellaratmosphere provision, the capability of treating non-synchronous rotation, and the generation of radial-velocity curves. Accuracy-related improvements were described by Wilson and Biermann ( $A A P$ 48,349). The procedures are now being generalized to include effects of orbital excentricity, with proper accounting for the variable tidal field on the figures of the components. Wilson further intends to incorporate the effects of radiation pressure and to develop programs for simultaneous solution of light and radial-velocity curves.

Comparisons of various methods on specific systems have been made in a number of cases. Binnendijk, in his paper referred to above (Vistas 21,359 ), gave parameters for V566 Oph, RZ Tau, and AW UMa. Results of other methods for these and other stars 
were reduced to the same set of parameters, thus making direct comparisons possible. The Russell-Merrill, Kitamura, Wood, and Wilson-Devinney procedures were used on CW Cas by Burchi et al. (ApSpSc 47,35) and on RT Per by Mancuso et al. (ApSpSc $47,277)$. For RZ Cas, Chambliss (PASP 88,22) obtained with the wood model elements which differ significantly from those found with the Russell-Merrill and KopalJurkevich methods. Chambliss and Leung (preprint) comparing the Russell-Merrill, Kopal-Jurkevich, and Wilson-Devinney approaches on SX Aur found the third procedure to yield the most consistent solution.

\section{Observational Data}

\section{A. Photometric Observations and Solutions (T. Herczeg)}

Tables 1 and 2 list publications reporting new photoelectric observations and orbital solutions. Photoelectric work continued to increase; among observatories that recently became very active in this field, we mention Athens, Biruni, Ege, Helwan, and Villanova. Quite frequently the number of observations are so high that their detailed publication becomes impractical, and the observations are deposited with one of the established repositories or made available upon request to the observer. Also, there are cases where publication is postponed to some later time, and only the observed light curves or other results are published presently. Table 1 contains papers that are obviously based on a "considerable amount" of new photoelectric data, thus, in contrast to previous practice, not only papers presenting individual observations. Satellite UV-photometry is included.

Novae at outburst are not regarded as belonging to the field of this Commission. The papers on V1500 Cyg quoted in Table 1 deal more or less with the periodic fluctuations in the light curve; if interpreted as an orbital phenomenon similar to those found in cataclysmic variables, they would suggest a very strong change of orbital period ( $c f$. Semeniuk et al., $A A$ 27,301).

Photographic studies are not listed in Table 1. Important ones are the optical histories of X-ray binaries: AM Her (Feigelson et al., ApJ 222,263), X Per (Gottlieb et al., ApJ 202,L13), V818 Sco (Wright et al., ApJ 200,171), and HD 245770 (Stier and Lilier, $\overline{A p J} \underline{206}, 257$ ), all derived from Harvard plates.

\section{B. Spectroscopic and Spectrophotometric Investigations (A. Batten)}

Table 3 lists spectroscopic and spectrophotometric studies, including determinations of orbital elements (references indicated by an asterisk). To save space, papers already cited in the Seventh Catalogue of the Orbital Elements of Spectroscopic Binary Systems (Batten et al., Publ DAO 15,121) are omitted.

Important fundamental work on detached main-sequence systems was continued by Popper and the Copenhagen group (especially Andersen). Popper extended his studies to include Algol-type systems, and reports the detection of the secondary $D-1$ ines in the spectra of a number of them, which should lead to better knowledge of their masses. A noteworthy individual achievement was the detection of the secondary spectrum of Algol itself by Tomkin and Lambert (ApJ 222,L119). Tomkin (ApJ 221,608) also detected the secondary spectrum of $\delta \mathrm{Lib}$. Early-type systems attracted considerable interest. Hutchings continues his work on mass loss and stellar winds. Conti and his associates published a number of critical re-investigations of systems containing O-type stars. Popper (ApJ $\underline{220}, \mathrm{LII}$ ) indicated that masses of some early-type systems - especially that of $\mathrm{V} 382 \overline{\mathrm{Cyg}}$, one of the most massive known - should be revised downwards. Wolf-Rayet binaries were the subject of considerable discussion at IAU Symposium 83; among others, Niemela reported on her continued work. A new study of $\theta$ Mus by Moffat and Seggewiss (AAp 54,607) should be particularly noted. Van Blerkom (ApJ 225,175) found supporting evidence that HD 45166 is binary, containing a lowmass star in Wolf-Rayet clothing. 
Table 1. Photoelectric Observations

RT And IBVS 1409, AB And Com Ankara 77, CN And IBVS 1087, 5 And Izv Krym 56,16, $\lambda$ And $A J$ 83,176, DX Aqr IBVS 1199, V337 Aql Per2v 20,375, v822 AqI ATs 956, V889 Aql $\overline{A A} 28$,221, RW Ara AAp Sup 26,227, SS Ari $\overline{A T s} 888$, UX Ari $A A \underline{27}, 281$, AJ 83,176, ApJ 225,919, SX Aur PerZv Pril 2, 51, IBVS 1278, WW Aur BAAS 7,463, CQ Aur RicA $\underline{8}, 555$, IU Aur $A A$ 28, 63, KR Aur Pis AZh 3,510, E Aur Publ Tartu 45, 284, TZ Boo IBVS 1086, 1487, AAp Sup 33,63, XY Boo AJ 82,648, AC Boo AAp Sup 29,57, $44 i$ Boo IBVS 1353, 1374, 1497, AAp Sup 32,361, SZ Cam Per2v 20,473, AS Cam IBVS 1090, ApSpSc 38,87, TX Cnc Obs 98,205, WY Cnc IBVS 1484, BAC 27,335, RS CVn BAAS 10,418, VZ CVn AAp 56,75, Rep Ege 225, AM CVn AA 25,371, BAAS 10, 419, R CMa AJ 82 ,51, UW CMa IBVS 1235, ApJ 220,582, CW CMa AJ 81, 1134, HH Car AAp Sup 22,263, $\overline{\mathrm{RZ}}$ Cas PASP 87,909, 88, 22, TV Cas AJ 82,740, BAC $\frac{12}{28}, 41$, AO Cas Pubi Tartu 43,103, BM Cas IBVS 1117, DO Cas AAp Sup 30,223, IBVS 1413, MN Cas AJ 81,665, SV Cen IBVS 1162, SZ Cen AAp 55,401, BH Cen ApJ 211,844, KT Cen AAp Sup 22,263, U Cep ATs 882, BAAS 9,352, ApJ Sup 31,1, ApJ 220, 251, 222,635, VV Cep Tokyo BuI2 247, 248, PASP 89,621, IBVS 1286, 1385, XY Cep BASI 4,83, CQ Cep Pis AZh 2,505 , CW Cep AAp Sup 25,151, EM Cep JRASC 69,307, ER Cep $M N$ 184,33, TX Cet AAP 70,355, XY Cet ApSpSe $\frac{38}{8}, 79$, RS Cha AAp Sup 33,87, RZ Cha AAp 44,343, RW Com BAAS $\underline{8}, 521$, CC Com PASP 88,777, ATs 918, Y Cyg RicA 8, 543, SS Cyg Pis A2h 2, 116, CG Cyg IBVS 1458, BAAS 9,623, MR Cyg AASin 18,68, MY Cyg AJ 80,976, V367 Cyg Pub2 Tartu 43,114, V388 Cyg AAp Sup 27,435, V444 Cyg PerZv 20, I, ApJ 221,193, V470 Cyg PASP 87,923, V1073 Cyg IBVS 1116, V1329 Cyg Per2v 20,345, v1341 Cyg = Cyg X-2 Pis AZh 4,402, Azh 53,511, Soob Zelenchuk 15,5, V1357 Cyg = Cyg X-1 MN 173, 15P, 177,63P, 182, 315, ApJ 205,855, BAAS 2,645, MittAG 43,225, Per2v 19,305, Nature 263, 393, v1500 Cyg AZh 54,583,592,600,620, AA 27,301, PASP 89,37,44, ApJ 211, L71, 225,954, AAp 55,171, 58,331, 31 Cyg AAp Sup 21,189, 32 Cyg AAp Sup 21,189, Tw Dra AAp Sup 32,57, BS Dra IBVS 1079, 1100, BY Dra ApJ 214,140, CM Dra ApJ 218,444, 71 Dra IBVS 1104, RU Eri BASI 4,83, CW Eri MittAG 38,231, U Gem ApJ 206,790, YY Gem Tokyo Bul2 240, AJ 83,618, X Gru AAp Sup 29,51, AK Her ApSpSc 52,387, AM Her ApJ 211, 859, 212, L113, 215,166, 219,597, 225, 542, 226,397, PASP 90,61, MN 183,73P, AAp 68, L1, Pis A2h 4, 183, Nature 271,335, DQ Her ApJ 196,L113, 207,195, HZ Her = Her X-1 PerZv 19,305, $\overline{\text { Pis Azh }}$ 1 No7,27, 4, 356, ApJ 208,567, I31, 4 Her AA 27,265, 88 Her BAC 29,278, TU Hor AAp 61,161, TT Hya BASI $\frac{1}{2}, 105$, VZ Hya ApSpSe 35,249 , AI Hya IBVS 1118, AAp Sup 33,103, HS Hya AAp 42, 303, $\chi^{\frac{2}{2}}$ Hya AAp Sup 31,307, RT Lac ApJ Sup 31,93, AJ 82,998, AR Lac PerZv 19,461, PASP 88,762, T's Shemak 37,9, XY Leo Obs 98, 205, AJ 83, 1452, AM Leo MittAG 43,186, T LMi PASJ 29,289, RR Lyn Ann Tokyo 16, 1, SW Lyn BAC 28, 120, B Lyr JAAVSO $\underline{4}, 18,80, \underline{5}, 16, A A p$ Sup 30,231, ApSpSc 41,121, $\overline{B A C} 27,215, M N$ 174, 217 , TY Men IBVS 1013, 1149, AAp 47,315, UX Men AAp 48, 49, AO Mon APSpSc 40,3, AR Mon ApJ 208,142, AU Mon IBVS 1348, V616 Mon AAp 48, 141, 50,445, ApJ 211, I15, IBVS 1173, Pis $A Z h$ 2, 112,402 , TU Mus AAp 45,107, $\theta$ Mus AAp 54,607, GM Nor AAp Sup 22,263, U Oph ApJ 214,423, BAAS 10,410, V502 Oph PerZv Prĩ 2, 161, V566 Oph $A N \underline{298}, 117$, PASP 88, 473, 89, 47, IBVS 1457, VV Ori BAAS 8, 306, BM Ori AA 26,91, n Ori IBVS 1418, $\theta^{l}$ Ori A IBVS 1238, 1274 , PASP 89,530, o Ori E Nature 262,116, ApJ 205, L87, MW Pav ApSpSC 46,155, U Peg IBVS 1010, X Per MN 176,217,225, AAp 61,47, ApJ 218, 504, IBVS 1359, $B \overline{A A} S$ 9, 349, RT Per ApSpSc 47,277, RW Per AA 28, 207, ST Per AA 26, 15, AG Per ApSpSc 57,17, BAAS 10,410, DM Per AAp Sup 25,291, IW Per BAAS 2,626, IZ Per BAAS 9,288, IX Per IBVS 1178, B Per AJ 81,57, 82,67, MN 172,235, 173 $, 271,180,461$, 184,523, PASP 87,745, AE Phe AAP Sup 24,399, AI Phe IBVS 1419, $\zeta$ Phe AAP Sup 23,261, RR Pic AAp 41,15, SZ PSC AJ 81,250, IBVS 1297, UV PsC IBVS 1381, V Pup $\overline{A A}$ 28,63, KX Pup AAp Sup 22, 263, NO Pup AAP 50,79, TY Pyx AA 28,231, IBVS 1489, U Sge PASP 88,688, UU Sge ApJ 223,252, WZ Sge ApJ 219,168, V1647 Sgr AAp 58,121, V523 Sco PASP 87,901, V818 Sco = Sco X-1 Soob Zelenchuk 15,5, A2h 53,511, AAp Sup 28,119, MN 176,91, $\sigma$ Sco IBVS 1432, RT Scl AAp Sup 28,389, RY Sct Bamberg Ver 121,386 , RZ Sct PASP 88,262, CV Ser ApJ 199,432, Af 11,49, Obs 97,76, AA 28, 55, RW Tau PASP 89,533, CD Tau ApSpSc 40,15, AJ 81,855, V471 Tau AAp 46, 197, ApSpSC 57,219, BAAS 10, 410, V711 Tau Ad 82, 47, 83, 176, ApJ 225,919, IBVS 1394, 1411, Obs 98, 207, X Tri AAp Sup 23,439, Byu 27 Abastuman 48, 13, RW Tri IBVS 1251, AQ Tuc

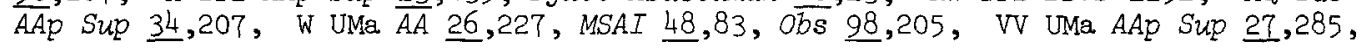


Table 1. (Continued)

XY UMa $A A \underline{27}, 273$, AN UMa BAAS 8,346, AW UMa AA 25,417, IBVS 1176, BAAS 10,410, RT UMi $A A p$ 29,333, RU UMi $A A \underline{27}, 187$, AO Vel IBVS 11l1, CV Vel AAp 58, 131, EO Vel AAp sup 22, $, 263, \gamma^{2}$ Vel AAp 57,151, AH Vir BAC 28,157, Obs 98,205, BF Vir $A A 26,253$, DL Vix $A \overline{A p}$ 61,107, ER VuI IBVS 1481, HR 3827A IBVS 1440, $\mathrm{HR} 4665$ IBVS 1432, HR 5110 IBVS 1459, HD 5980 PASP 90,101, HD 13970 AAp 10,105, HD 20301 IBVS I317, AAp Sup 29,313, HD 38735 AAp Sup 29,313, HD 47732 ApJ 222,574, HD 60168A AAp Sup 29,313, HD 71581 AAp Sup 29,313, HD $77581=$ Vel X-1 AAp 55, 473, AAp Sup 27, 433, $\overline{M N}$ 183,813, HD 86441 IBVS 1265, HD 93206 IBVS 1265, HD 128141 PASP 87, 877, HD 152235 IBVS 1265, HD $153919=3$ U 1700-37 AAp 52,139, 54,543,683, 58, L5, HD 159176 BAAS 7,533, HD 162724 ApJ 201,792, HD 173198 IBVS 1041, 1306, HD 193793 MN 185, 467, HD $19 \overline{9} 497$ IBVS 1214, HD $22 \overline{4085}$ PASP 89,280, HDE 271213 ApJ 207, 329, HDE 271227 ApJ 207,329, BD $-3^{\circ} 5357$ PASP 89,616, BD $-7^{\circ} 3007$ PASP 87,716, CD -33 12119 PASP 89,720, CD $-42^{\circ} 14462$ ApJ 214, 471, LB 3459 MN 183,523, IS 55 -8 PASP 90, 191, PG 1413+01 ApJ 224,892, NGC 2264 W92 PASP 89,874, Sk $160=$ SMC X-1 AAP 54, 307, $A A p$ Sup 29,339, Wray $977=3 \mathrm{U} 1223-62$ ApJ 203,689, AAp 49, 321, 54, 733, MittAG 43, 227, Cir X-1 $M N$ 183,335, Cyg X-3 ApJ 207,78, LMC X-4 AAp 29, I9, 4U I626-67 ApJ 225,1001.

Two objects continuing to attract much attention are $\beta$ Lyr and VV Cep. Hack et a2. (ApJ Sup 34,565) presented evidence for the secondary spectrum in the far UV that would set an upper limit of 3:1 (secondary:primary) for the mass-ratio. On the other hand, Dadaev (Izv Pulkovo 193,41, 194,18) claims to have found the secondary spectrum in the photographic region and gives the mass-ratio 1.1:1. Dadaev (Izv Pulkovo 195,98, and subsequent papers) is now studying the emission-line spectrum and relating it to the pattern of gas streams. Batten, Ringuelet, and Sahade continue their studies of emission lines. The other system, VV Cep, underwent eclipse during the triennium. Wright now has high-dispersion spectrograms covering a complete orbital cycle. He published orbital elements and a discussion of gas streams (JRASC 11,152), and continued observations through eclipse. For a third famous star, $\varepsilon$ Aur, IUE observations indicated the presence of a hot companion (Hack and Selvelli, Nature 276,376).

Binaries containing intrinsic variables were studied by several groups. Abt and Levy (PASP 90,188) drew attention to the probable duplicity of the cepheid $Y$ Oph; and the dwarf cepheid RS Gru was shown to have a companion by Balona and Martin (MN 184, 1). Rodono (AAp 66,175 ) studied flare-active systems. The RS CVn group of binaries naturally attracted much attention, because of the discovery that they are radio sources. The study of optical counterparts of $X$-ray binaries continued. New identifications were made and attempted, and orbital elements of known binaries were refined (Conti, AAp 63,225; Hutchings, ApJ 226,264).

Orbital elements of several multiple systems were determined, especially by Fekel, who is continuing his work. Geyer and Patkos report that ADS 12019 is quadruple (component $A$ is the eclipsing variable BH Dra). Morbey et al. (PASP 89,851) showed that one component of ADS 11060 is a close binary. The visual pair has now been followed through periastron and is the spectroscopic binary with the most excentric orbit known $(e=0.96)$. Radial velocities of other visual binaries have been measured, especially $\delta$ Equ (Popper and Dworetzky, PASP 90, TI; Scarfe et al., in press).

\section{Polarimetric Studies (A.M. Cherepashchuk)}

"Ordinary" close binaries show variable linear polarization due to scattering from circumstellar matter, and possibly to some degree also from other mechanisms. Pfeiffer and Koch (PASP 89,147), reviewing existing data (in many cases obtained by the authors themselves) found that, with the exception of $U$ Oph, unevolved binaries do not show intrinsic polarization. Phase-locked polarization was detected in $A O$ Cas (Pfeiffer, AJ 81,1000; Rudy and Kemp, ApJ 207,L125), u Her (Rudy and Kemp, ApJ 216,767), HD 47129 (Rudy and Herman, PASP $\underline{90}, 163$ ), and in AIgoI, U Sge and V444 Cyg 
Table 2. Photometric Solutions

RT And AAp Sup 32,351, FK Aql Trudy Kazan 41,3, v805 Aq1 AAp Sup 32,351, o Aq1 AAp Sup 33,91, RW Ara AAp Sup 26,227, V535 Ara ApJ 222,917, RX Ari BAC 26, 257, RZ Aur Trudy Kazon 41,3, TT Aur AAp Sup 33,91, WW Aur BAAS 1,463, AAp Sup 33,91, AR Aur $A A p$ Sup 33,91, IU Aur $A A$ 28, 63, LY Aur AAp 62,291, ZZ Boo AAp Sup 32, 351 , AC Boo ApSpSc 29,57, AAp 63,193, 44i Boo AAp Sup 32,361, S2 Cam ApSpSc 46, 407, AS Cam ApSpSc 38,87, AY Cam ApSpSe 46,261, S Cnc Tmidy Kazan 39, 42, ApSpSc 47,375, RZ Cnc ApJ 208,142, AAp 61,469,809, TX Cnc Obs 98,205, RS CVn BAAS 10,418, VZ CVn AAp 56,75, Rep Ege 225, R CMa AJ 82,51, UW CMa ApJ 220,582, 222,924, MN 185, 485, CW CMa $A J$ 81,1134, HH Car AAp Sup 22,263, RZ Cas PASP 88,22, TV Cas ATs 881, BAC 28, 41, YZ Cas ApSpSc 56,389, 57,71, AO Cas ApJ 223,202, CW Cas ApSpSc 47,35, DO Cas AAp Sup 30,223, MN Cas AJ 82,290, V523 Cas ATs 873, RR Cen MittAG 38,225, SV Cen PASP 88, $244, M N 176,625$, SZ Cen AAp 55,401, BH Cen ApJ 211,844, KT Cen AAp Sup 22, 263, U Cep $\overline{B A A S}$ 9,352, AAp 61, 469, VW Cep Publ Tartu 43,130,176, WX Cep $A A p$ Sup 32,351, XY Cep BASI 4,83, ZZ Cep AAp Sup 33,91, $\overrightarrow{A H}$ Cep AAp Sup 33,91, CW Cep $\overline{A A p}$ Sup 25,151, 33,91, EI Cep Ann Tokyo 16,37, AAp Sup 32, 351, EK Cep ATs 971, ER Cep MN 184, 33, SS Cet Trudy Kazan 41,3, TX Cet AAp 70,355, XY Cet ApSpSc 38,79, 56,293, RS Cha BAC 26,257, AAp 44,459, RZ Cha AAp 44, 343, CC Com PASP 88,777, U CrB AAp 61, 469, RV Crt IBVS 1272, SW Cyg Trudy Kazan 41, 3 , GG Cyg BAAS 2,623, MR Cyg AASin 18,68, AAp 66,161, AAp Sup 33,91, Vizlanova Contr 1, MY Cyg BAC 27, 125, AJ 80,976, V367 Cyg Pubz Tartu 43,114, V382 Cyg AAp Sup 33,91, V388 Cyg AAp Sup 27, 435, v444 Cyg ApJ 221,193, v453 Cyg BAAS 9,352, AAp Sup 33,91, V729 Cyg ApJ 224,565, V1073 Cyg ApJ 222,917, V1357 Cyg = Cyg X-1 MittAG 43, 225, ApJ 226,264, RR Dra Trudy Kazan 41,3, TW Dra AAp Sup 32,57, CM Dra ApJ 218, 444 , 71 Dra IBVS 1104, RU Eri BASI 4, 83, AS Eri AAp 62,291, CW Eri MittAG 38,231, RW Gem ApSpSc 46,261, YY Gem Tokyo BuZ2 240, AJ 83,618, X Gru AAp Sup 29,51, RX Her AAp Sup 33,91, TX Her AAp Sup 32,351, AK Her ApSpSc 47,79, 52, 387, BAAS 10,411, $\mathrm{HZ}$ Her $=\mathrm{Her} \mathrm{X}-1$ ApJ 208,567 , V338 Her BAC 26, 257, u Her AAp 61, 469, 66,161, VZ Hya ApSpSe $35,249, A A p$ Sup 32,351 , AI Hya AAp 66, 377, HS Hya AAp 42, 303, $x^{2}$ Hya $A A p \underline{67}, \frac{315}{15}$ RT Lac ApJ Sup 31,93, AJ 82,998, AR Lac PASP 88, 762 , ApSpSc 52,213, Ts Shemak 51-52,20, CM Lac BAAS 8,305, AAp Sup 32,351, UV LeO BAC

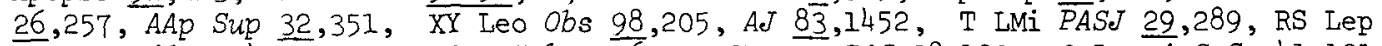
Perzv Prit 3,149, RR Lyn Ann Tokyo 16,1, SW Lyn BAC 28, 120, в Lyr ApSpSc 41,121, UX Men AAp 48,49, RW Mon Trudy Kazan 41,3, TU Mon AAp 61,469, AO Mon ApSpSc 40,3,

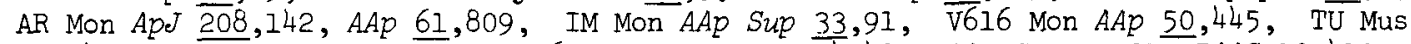
AAp 45,107, GM Nor AAp Sup 22,263, U Oph ApJ 214, 423, AAp Sup 33,91, BAAS 10, 410, WZ oph AAp Sup 32,351, V566 Oph Vistas 21,359, PASP 88,473, 89, 366, V1010 0ph ApJ 211,853, WV Ori AAp Sup 33,91, $\theta^{1}$ Ori A IBVS 1129, MW Pav ApSpSc 46, 155, AW Peg AAp 62,291, RT Per ApSpSc 47,277, 58,3, RW Per AA 28,207, RY Per Trudy Kazan 41,3, ST Per AA 26,15, AG Per ApSpSc 57,17, BAAS 10,410, DM Per AAp Sup 25,291, IZ Per BAAS 9,288, B Per PASP 87,745, AJ 81,57, 82,67, ApSpSc 47,459, 51, 265, 5 Phe AAp 46,205, SZ Psc AJ 81,250, V Pup AAp $61,275,469, A A \underline{28}, 63$, AU Pup ApJ 222,917, $\frac{1}{\mathrm{KX}}$ Pup AAp Sup 22, 263 , No Pup AAp 50,79, U Sge PASP $\underline{88}, 688$, ApSpSc 47, $361, \underline{56}, 219$, $A A p$ 61,469, UU Sge ApJ 223,252, WZ Sge ApJ 219,168, V356 Sgr AAp 61,469, V1647 Sgr AAp 58,121, v523 Sco PASP 87,901, V7O1 sco AAp 61,137, $\mu^{\top}$ Sco AAp 61,469, RZ Sct PASP 88, 262, RW Tau Tav Ehngelg 41-42,196, ApSpSe 47, 361, RZ Tau Vistas 21,359, CD Tau Ad 81,855, ApSpSc 40,15, V471 Ta12 AAp 46, 197, $\lambda$ Tau AAp 62,291, X Tri AAp Sup 23, 439, Byuz2 Abastuman 48,13, AQ Tuc $\overline{A A p}$ Sup 34,207 , W UMa

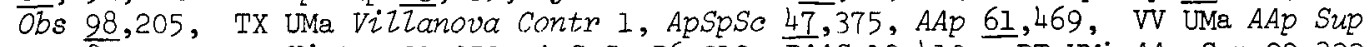
27,285, AW UMa Vistas 21,359, ApSpSe 56,219, BAAS 10,410, RT UMi AAp Sup 29,333, RU UMi $A A$ 27, 187, CV Vel AAp 58,131, EO Vel AAp Sup 22,263, AH Vir Obs 98,205 , $B F$ Vir $A A \underline{26}, 253$, DL Vir AAp 61,107, Z VuI AAp 61, 469, RS Vul AAp 61, 469, BV845 PASP 90,97, HD 47732 ApJ 222, 574 , HD $77581=\mathrm{Vel} \mathrm{X}-1$ Pis AZh 1 Nol2,13, ApJ 202, L131, 226, 264, AAP 54, 167, HD 101799 PASP 88,936, HD $153919=3 \mathrm{U} 1700-37 \mathrm{ApJ}$ 226,264, HD 159176 BAAS 1,533, HD 162724 ApJ 201,792, HD 173198 IBVS 1306, LB $3459 M N \underline{183}, 523$, PG 1413+01 ApJ 224,892, Sk $160=$ SMC X-1 ApJ $204,551$. 
Table 3. Spectrographic Observations

$\zeta$ And Izv Krym 56,16, $\lambda$ And BAAS 8,353, PASP 88,137, Nature 275, 389, $v$ And ApJ Sup 36,241*, O And ApSpSo 46,379, v5999 Aql Izv Krym 58,56, v60 3 Aql BAAS 9,627 , UX Ari IBVS 1075, ApJ 216,503, 225,919, AJ 83,795, MN 182,77, k Ari AAp Sup 27,35, 35 Ari ApJ Sup 36,241*, EO Aur ApJ 220,L11, KR Aur Pis AZh 3,271,510, a Aur Nature 275,389, $\varepsilon$ Aur ApSpSc 49,179, Nature 276,376, $\zeta$ Aur ApSpSc 36,273, T2 Boo AAp Sup 33,63, ZZ Boo AAp Sup 32,347*, a Cam ApJ 223,908, S Cnc Obs 96,9, RS CVn $A A$ 26,301, ApJ 216,503, MN 182,77, TX CVn AAp Sup 34,211, UW CMa ApJ 208,760, EZ CMa $\overline{A f} 12,180, \overline{S X}$ Cas ApJ $209,821, \xi$ Cas ApJ Sup $36,241 *$, o Cas ApJ Sup $36,241 *$, B Cen $M N$ 172,639, U Cep ApJ 209,821, 212,446, VV Cep AAp 46,317, 64, 253, EM Cep Izv Krym 56,11, a CrB ApSpSc 46,379, SS Cyg Af Iss2 8,3, Nature 275, 385, V367 Cyg ApSpSe 53,345, V382 Cyg ApJ 220,LII, V389 Cyg AAp 68,437*, V453 Cyg ApJ 220,LII, V478 Cyg ApJ 220,L11, V1329 $\overline{\mathrm{Cyg}}$ Bamberg Ver 121,383, V1341 Cyg $=\mathrm{Cyg} \mathrm{X}-2 \mathrm{Pis} A 2 \mathrm{~h}$ 2,534, ApJ 207,L171, V1357 Cyg = Cyg X-1 ApJ 213,815, BAAS 9,645, Nature 275, 400, V1500 Cyg PASP 89,37, ApJ 207,L41, 211,184, 217,775, 224,899, AAp 56,181, 9 Cyg AJ 83,615*, $57 \mathrm{Cyg}$ ApJ Sup 36,241*, AI Dra $A A \underline{28}, 41 *$, BY Dra PASP 89,69, CM Dra $A A$

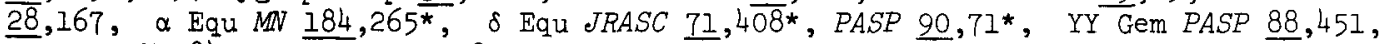
RS Gru MN 184,1, $\bar{Z}$ Her $M N$ 182,77, AD Her PASP 90,312*, AM Her ApJ 212, L117, 218,L121, $\overline{\text { PASP } 89,374, \mathrm{HZ}} \overline{\mathrm{Her}}=\mathrm{Her} \mathrm{X}-1$ AAp 59, 441, ApJ 209,547, 222, L33, PASP 89,285, Nature 275,400, v600 Her AAp 51,1, 1 Her ApJ Sup 36, $241, \frac{2}{88}$ Her IBVS 1496 , TU Hor AAp 61,161, RT Lac IBVS 1130, AR Lac PerZv 19,377, 20,207, Ts Shemak 35,8,11, 36,11, ApJ $\underline{216}, 503,508, M N 182,77,5$ Lac PASP 90,184*, T LMi PASJ 29, 289, FX Lib $\overline{A A p}$ Sup 34, $51, \delta$ Lib ApJ 221,608*, RR Lyn Ann Tokyo 16, 1, B Lyr Izv Pulkovo 193, 41, $194,18, \overline{A Z h} 52,710$, Ts Lvov 51,20, ApSpSc 38,353, ApJ 는 $, 777, \underline{208}, 468, \underline{210}, 853, A A p$ 50,335, Af Iss 2 9,3, PASP 88,899, ATs 966, Izv Krym 58,75, n Lyr ApJ Sup 36,241*,

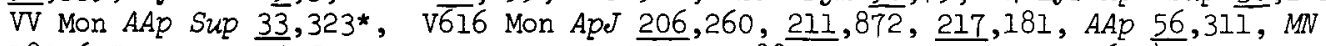
180,657, U Oph ApJ 220,LII, Y Oph PASP 90,188*, к Oph ApJ Sup 36, 241*, BM Ori

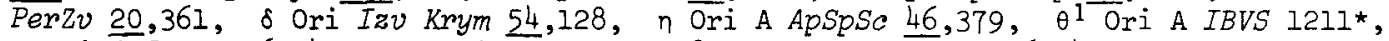
$\xi$ Ori ApJ Sup 36,241*, o Ori E ApJ 205,L87, $\psi$ Ori ApJ Sup 36,241*, 22 Ori ApJ Sup 36,241*, 64 ori BAAS 2,623*, AG Peg BAAS 10,410, K Peg PASP 89,857, X Per PASP 88,754, MN 176,233, 181,685, Izv Krym 51,45, IBVS 1461, LX Per MN 182,77, B Per $\overline{M N}$ 176,5P, IBVS 1312, ApJ 222,L119*, 20 Per PASP 90,297, AE Phe AA 28, 49*, RR Pic BAAS 9,627, SZ Psc MN 182,77, V Pup ApJ 210,143, VV Pup ApJ 225,201, U Sge ApJ 209,821, 4l Sex A Ad 83,303*, V818 Sco $=$ Sco X-1 ApJ 206, L49, BAAS 9,627, RY Sct Bamberg Ver 121,386, W Ser IBVS 1482, V711 Tau AJ 81,771*, 83,795, ApJ 225,919, BAAS 9,624, Nature 275,389, \& Tau AJ 82,176*, $\theta^{1}$ Tau AJ 82, 176 , $\tau$ Tau ApJ Sup 36,241*, 51 Tau BAAS 9,600*, RR Tel $\overline{M N} 182,57 \mathrm{P}$, RW Tri $\overline{B A} A S$ 9,556, $\alpha$ Tri $A A$ $\frac{28}{36}, 235^{*}, \quad \gamma^{2}$ Vel $M N 17 \overline{6}, 29 P$, ApJ Sup 36, $2 \overline{17}, \alpha$ Vir ApSpSe 46,379, I Vul ApJ Sup $\underline{36}, 241 *, 15$ Vul Obs $\underline{97}, 2$, HR 1034 ApJ Sup 36,241*, HR 1970 Obs $98,122 *$, HR 2013 Obs 96,54, HR 2172 AAP Sup 27,31, HR $2317 \overline{O b s ~ 98,232 *, ~ H R ~} 3080$ ApJ 203,435, HR 4665 BAAS 10,419, HR 5049 AAp 58,93, HR 6387 Obs $98,14 *$, HR 6902 AJ $83,615 *$, HR 7083 Obs 97,235*, HD 698 PASP 90,179*, HD 5303 IBVS 1342 , HD 28033 AJ $83,1114 *$, HD 30738 AJ $\underline{83}, 1114 *$, HD 47732 ApJ 222,570*, HD 50896 ApJ Sup 36,217, HD 67084 PASP 90,204*, HD 67198 PASP 90,204, HD $77581=\mathrm{Vel} \mathrm{X}-1$ AAp Sup 30,195*, MittAG 43,219, HD 92740 ApJ Sup 36, 217, AAp 70,69, HD 135774-5 AJ 83,615*, HD 137569 $\overline{B A A S} 9,351^{*}$, HD 147508 obs 98,47*, HD 150958 ApJ 225, 165, HD 152270 MittAG 43,148, HD $15 \overline{3919}=3 \mathrm{U}$ 1700-37 AAp 47,19, 56,433, 64,399*, AAp Sup 32,375, ApJ 217, I35, Nature 275,400, HD 155989 Obs 98, 158*, HD 165052 ApJ 224,558*, HD 167771 ApJ 224, 558*, HD 183030 Obs 98,118*, HD 187399 Af 12,623, HD 188753 Obs 98,122*, HD 190918 JRASC 71,407*, HD 204934 Obs 98,122*, HD 214419 Kodaikanal Bul2 2,89, HDE 245770 AAp 56,311, ApJ 215,568, 223,530, BD +27 4642 Izv Krym 57, 31, BD +24.692 AJ 83, 1114*, $\overline{\mathrm{BD}}-35357$ PASP 89,616 , BD $-7^{\circ} 3007$ PASP 89,716*, ADS 14893 B ApJ 205, 194*, Feige 24 ApJ 223,260*, Gliese 815 AJ 83,1445*, Wray $977=3 \mathrm{U} 1223-62 \mathrm{Mi} t t A \bar{G}$ 43 $, 227^{\star}, \mathrm{G} 61-29$ $A A$ 25,227, CRL $2104 M N$ 185,47, CRL 2179 MN 185,47, w Cen V78 BAAS 10,411, NGC 1851 UV5 AAp 59,L23, SMC X-2 ApJ 223,L79, SMC X-3 ApJ 223, L79, LMC X-4 AAp 59,L9, ApJ 225,548*, 4U 1538-52 ApJ 225, L63, 3U 1728-24 ApJ $\underline{211}, 866$. 
(Rudy and Kemp, ApJ 221,200). These observations allow determinations of orbital inclinations (Rudy and Kemp, ApJ 221,200; McLean, Obs 98,205). Tapia (ApJ 212,I125) made the exciting discovery that the radiation from $A M$ Her exhibited high linear and circular polarization in the $V$ and $B$ wave-length bands, indicating a magnetic field of about $2 \times 10^{8}$ gauss for the white-dwarf component. Further observations were published by Michalsky et al. (ApJ 216,L35), Stockman et al. (ApJ 217,815), Bailey et al. (MN 183,73P), and Priedhorsky et al. (ApJ 225,542). Similar polarization properties were found for AN UMa (Krzeminski and Serkowski, ApJ 216, L45; Downes and Urbanski, PASP 90,458) and for VV Pup (Tapia, IAUC 3054). In a search for other "polars", Vojkhanskaya et al. (Pis $A Z h 4,272$ ) picked out MV Lyr as a promising candidate. X-ray binaries with harder spectra than AM Her show only weak intrinsic polarization in the optical region. For Cyg X-I Michalsky and Swedlund ( $A p J \underline{212}, 221$ ) reported slight variations of the circular polarization synchronous with the orbital period, $5.6 \mathrm{~d}$, but this periodicity was not apparent in 1977 (Michalsky et al., ApJ 225,599). For the linear polarization Kemp et al. found a period of 39.2 d from $\mathrm{UV}$-data (Nature 270,227) and, from an extensive set of observations, orbital synchronism at long wave-lenghts (ApJ 220, L123). Long-term variations are probably present.

\section{X-ray Observations ( $Y$. Kondo)}

X-ray satellites that have been operational during the triennium and used for observations of close binaries are listed below (with launch dates and experimenters): Copernicus 1972 University College London

Ariel-5 (UK-5) 1974 Mullard Space Sci. Lab., Univ. of Leicester, Imperial College, Goddard Space Flt. Ctr., Appleton Lab.

SAS-3 1975 M.I.T.

OSO-8 1975 Columbia Univ., Goddard Space Flt. Ctr., Lockhead, Univ. of

HEAO-1 1977 Goddard Space Flt. Ctr., C.I.T., Univ. of Calif. Berkeley, Naval Res. Lab., Ctr. for Astrophysics, M.I.T., Univ. of Calif. San Diego

HEAO-2 1978 Goddard Space Flt. Ctr., Ctr for Astrophysics, M.I.T., Columbia Univ.

In addition, rocket experiments were flown to observe selected X-ray binaries, e.g. by Boldt and co-workers. Balloon-borne experiments also produced interesting new results, e.g., Trümper and co-workers.

Some 40 galactic X-ray sources have now been identified with optical counterparts, the great majority being close binaries. An informative review on positions and identifications was given by Bradt et al. at the COSPAR/IAU Symposium on $X$-ray Astronomy, held at Innsbruck 1978. Reference may also be made to the series of letters. to Nature $(\underline{269}, 112, \underline{270}, 586, \underline{271}, 225, \underline{272}, 701, \underline{273}, 364)$ by the SAS-3 team. As the number of optical identifications increases, it is possible to segregate different classes of objects. One classification scheme is the following:

A. Sources comprising compact object (white dwarf, neutron star, black hole) and early-type star (often supergiants). Examples: SMC X-1, Vel $\mathrm{X}-1$, Cen $\mathrm{X}-3, \mathrm{Cyg} \mathrm{X}-1$, V861 Sco, $4 \mathrm{U} 1700-37$.

B. Compact object with late-type companion: HZ Her, AM Her, Sco X-1, Cyg X-3.

C. Dwarf novae: SS Cyg, U Gem, EX Hya.

D. RS CVn systems: V7II Tau, UX Ari, RS CVn.

E. Other transient X-ray sources - novae, X-ray novae, etc.: V616 Mon, A 0535+26.

$F$. Other binaries identified as X-ray sources: Algol, Sirius, Capella, $\gamma$ Cas.

G. X-ray bursters: Several bursters have been identified with stellar objects, making close-binary models most probable. Many bursters appear associated with globular clusters.

There are additional optically identified objects that can be fitted into some of these categories; the ones above are merely examples. As more optical identifications are made, additional classes of sources may be found. Of course, a variety of other classification schemes are possible. 
Publications on X-ray emitting binaries are very numerous, and only some few items can be mentioned within the limited space available. New binary orbits from pulse-phase data were determined for Cen X-3 (Fabbiano and Schreier, ApJ 214,235), Vel X-1 (Rappaport et al., ApJ 206, L103; Charles et al., MN 183,813), SMC X-1 (Primini et al., ApJ 210,L71, 217,543), 4U 0115+63 (Rappaport et al. , ApJ 224, LI), 3U 1223-62 (White et al., MN 184,67P), and 3U 1538-52 (Davison et al., MN 181,73P). Trümper and his co-workers $(A \overline{p J} 219, \mathrm{L105})$ discovered in the spectrum of Her X -1 a line feature at about $58 \mathrm{keV}$, interpreted as cyclotron emission. Iron lines are reported by many groups in the spectra of several X-ray binaries. Observations of the $\gamma$-ray flux from Cyg X-3 were discussed by Vladimirsky et al. (Izv Krym 58,44).

\section{E. Radio Observations (D.M. Gibson)}

Radio surveys of binaries from one or more of the classes below were reported by Altenhoff et al. (AAp 46,11), Feldman (BAAS 10,418), Gibson (Thesis, UVa), Owen and Gibson ( $A J$ Dec. 1978), Spangler et al. ( $A J \overline{82}, 989$ ), Woodsworth and Hughes (AAp 58,105), and Wright and Allen (MN 184,893).

RS CVn and related binaries. Detected RS CVn systems include UX Ari, RT Lac, AR Lac, SZ Psc, TY Pyx (probable), V7ll Tau (HR 1099), HD 224085 (Owen and Gibson, AJ Dec. 1978), and HR 5llo (Feldman, pri. com.). Emission from the related longperiod systems $\lambda$ And (Bath and Wallerstein, PASP 88,759), and $\sigma$ Gem, $\sigma \mathrm{CrB}$, HD 216489, and the short-period system UV PSc (Spangler et al., AJ 82,989) was also reported. Algol and $b$ Per can probably be regarded as similar objects. The above sources are highly variable - from about $10^{16}$ to $10^{19} \mathrm{erg} \mathrm{s}^{-1} \mathrm{~Hz}^{-1}$ (Gibson et al., AJ Dec. 1978; Feldman et al., AJ Dec. 1978; Gibson et al., PASP Nov. 1978) - as befits their compact size (few $R_{\odot}$; Clark et al., ApJ 206,Ll07; Owen and Spangler, ApJ 217,L41). The gyrosynchrotron emission (Owen et al., ApJ 210,L27) arises just beyond the X-ray coronae (Gibson et al., AJ Dec. 1978), and thus their spectra can be affected by either self-absorption (Owen et al., ApJ 210,L27) or thermal absorption (Spangler, AJ 82,169). The circular polarization can be quite large (74\%; Mutel and Weisberg, $A J$ Dec. 1978) and has been observed to be rapidly variable (Brown and Crane, AJ Dec. 1978). The emission is associated with "super solar-flare" phenomena intrinsic to one of the late-type (subgiant) components (Weiler et al., ApJ 225,919), not with mass exchange. However, binarity - particularly faster rotation, larger differential rotation, and smaller surface gravity relative to single stars (Hall and Shore, BAAS 10,418) - seems to enhance their radio emissivity relative to their single counterparts (Gibson, BAAS $\underline{9}, 600$ ).

$X$-ray/radio binaries. Continued observations of Cyg $X-1$ (Hjellming and Gibson, in prep.), including some correlated with X-ray bursts (Hjellming, $X$-ray Binaries, NASA SP-389, p. 495; Braes and Miley, Nature 264,731), now indicate the emission is non-thermal, but the spectrum is probably affected by the 0-star's wind (Johnson et al., in prep.). Hjellming and Helfand (in prep.) and Hjellming (ApJ 22l,225) reconfirmed the identifications of $\mathrm{Cyg} X-2$ and GX $17+2$ with G-type stars. Further observations of Cyg X-3 (McEllin, MN 175,5P; Mason et al., ApJ 207,78; Ledden et al., Nature 252,669) were useful in determining conditions in the emission region both during outbursts (Seaquist, ApJ 207,88; Shields and Wheeler, ApL 17,69; Woodsworth and Hughes, ApJ 208,863) and quiscent periods (Seaquist and Gregory, ApL 18,65). Several new types of X-ray/radio binaries were detected, including the highly unusual counterpart to $\mathrm{Cir} X-1$ (Whelan et al., MN 181,259; Thomas et al., MN 185,29P). Nonthermal radio counterparts to the transient sources A 0620-00 (Owen et al., ApJ 203,L15; Little et al., Nature 261,113) and one near the galactic centre (Davies et at. Nature 261, 476) were also detected. The controversy over the nature of the globular-cluster transients (cf., Smith, Nature 261,453) or whether they have even been detected at radio frequencies (Johnson, ApJ $\underline{208}, 706$ ) remains unresolved.

Novae. While radio emission from V1500 Cyg was apparently weak and short-lived (Pynzar et al., ATs 893; Altunin, Pis AZh 2,,299; Hjellming, Bamberg Ver 121,279), 
emission from $F H$ Ser and $H R$ Del is still being monitored (Hjellming and Vandenberg, in prep.). A thick inhomogeneous shell model for $\mathrm{FH}$ Ser was developed by Seaquist and Palimaka (ApJ 217,781 ).

Wolf-Rayet binaries and symbiotic stars. Variable thermal emission from WRbinaries was detected (Florkowski and Gottesman, IBVS 1101), with $\gamma^{2}$ Vel (Seaquist, ApJ 203,L35) and HD 193793 (Florkowski and Gottesman, $M N 179,105$ ) receiving continuing monitoring, the latter in conjunction with a recent IR-outburst. Variable thermal emission associated with recent outbusts was monitored for the symbiotic objects V1016 Cyg (FitzGerald et al., BAAS 8,372), AG Peg (Gregory et al., ApJ 211,429 ), and HM Sge (Feldman, JRASC 11,386 ).

Monitoring of the thermal-type, compact stellar-wind binaries CC Cas and B Lyr continued (Gibson and Newell, in prep.). Monitoring of a Sco resulted in detection of both $A$ and $B$ components (Gibson, in prep.). The latter's spectrum, while nonthermal, is varying, which suggests it is either a coherent emitter or has an unusual particle-acceleration mechanism. Observations of the binary pulsar, PSR $1913+16$, continued; a recent progress report was given by Fowler and Taylor (BAAS 10,447$)$.

\section{Physical Data}

\section{A. Absolute Dimensions ( $T$. Herczeg)}

New determinations of absolute dimensions are listed in Table 4. A novel feature here is the fairly extensive rediscussion of earlier light-curves by applying modern programs of analysis and then using existing spectrographic data to obtain revised absolute dimensions. A considerable part of this work was done by Cester and his collaborators with Wood's WINK program. Leung, Schneider, and Wilson, in several papers, systematically rediscussed the "W UMa-like" light-curves of early, massive contact (or near-contact) systems. Here the Russell-Merrill method is clearly inadequate; they used the Wilson-Devinney code and found in several cases various degrees of over-contact between the components.

\section{B. Period Changes (T. Herczeg)}

Some few theoretical investigations continued the work toward establishing a relationship between period changes and the modes and rates of mass transfer and mass 1oss (Amnuel and Guseinov, AAp 54,23; Chau, ApJ 219,1038; Mukhametkalieva and Omarov, Tmidy Alma-Ata 28,24; Piotrowski and Ziolkowski, $A A$ 28,295). Several detailed evolutionary studies touched upon period changes in connection with the time scales involved. In general, however, we are still rather far from being able to use observed period changes as checks on evolution theories. One reason may be the apparently erratic behaviour of the periods, short-term fluctuations exhibited by a surprising number of systems. Also, the time scale is in most cases perhaps too long for the only 30 - 40 years of more systematic and accurate observations, with the possible exception of a few objects in rapid evolution, such as B Lyr and SV Cen. This, of course, emphasizes the importance of collecting as many well-defined minimum epochs as possible, for the benefit of future studies.

At IAU Colloquium 42, Kreiner (Bamberg Ver 121, 393) reviewed period changes of W UMa stars, and Smak (Bamberg Ver 121,365) discussed secular mass-loss and period changes of cataclysmic variables. Of the many individual systems observed and discussed during the triennium, only a few particularly interesting ones will be mentioned. In the case of XY UMa, Geyer (ApSpSc 48,137) demonstrated the occurrence of spurious changes due to light-curve anomalies. Hall et al., AJ 81,1138 ) discussed the "migrating wave" in the light curve of AR Lac and its relation to observed period changes; they concluded that although a correlation exists, the period changes are almost certainly real. Other studies of AR Lac are by Babaev (IzV AN AzSSR 4 , 3), Chambliss (PASP 88,762), and Theokas (ApSpSe 52,213). A detailed study of U Oph by 
Table 4. Absolute Dimensions

RT And AAp Sup 32,351, v805 Aql AAp Sup 32,351, o Aql AAp Sup 33,91, TT Aur AAp Sup 33,91, WW Aur BAAS 1,463, AAp Sup 33,91, AR Aur AAp Sup 33,91, IU Aur AAp 59,9, LY Aur AAp 62,291, E Aur AAp 69,23, zZ Boo AAp Sup 32, 347, 33, 91 , WX Cep AAp Sup 32,351, AH Cep AAp Sup 33,91, CW Cep AAp Sup 33,91, EI Cep AAp Sup 32,351, SZ Cam ApSpSc 36,329, AS Cam ApSpSc 38,87, RZ Cnc ApJ 208,142, AAp 61,469, UX CVn AAp 70,451, UW CMa PASP 89,668, ApJ 220,582, 222,924, MN $\underline{185}, 485$, RZ Cas PASP 88,22, TV Cas ATs 881, BAC 28, 41, AO Cas Apd 223,202, AZ Cas PASP 89,882, V523 Cas ATs 873, SV Cen PASP 88,244, MN 176,625, SZ Cen AAp 45, 203, 55, 401, BH Cen ApJ 211,844, U Cep AAp 61,469, VV Cep JRASC 71,152, CW Cep AAp Sup 25,151, EI Cep Ann Tokyo 16,37, EK Cep ATs 971, ER Cep MN 1 $144,33, \xi$ Cep PASP 88, 944 , XY Cet ApSpSc 38, $\overline{79}, 56,293$, 13 Cet PASP 88,50, RS Cha AAp 44, 445, RZ Cha AAp 44,343,349, CC Com PASP 89,684, U CrB AAp 61,469, MR Cyg AAp 66, 161, AAp Sup 33,91, $\overline{\mathrm{MY}} \mathrm{Cyg} B A C$ 27, 125, V382 Cyg AAp Sup 33,91, V444 Cyg ApJ 221,193, v453 Cyg AAp Sup 33,91, V729 Cyg ApJ 224,565, V1073 Cyg ApJ 222,917, AI Dra AA 28,41, CM Dra ApJ 218,444, $\delta$ Equ PASP 90,71, AS Eri AAp 62,291, CW Eri MittAG 38,231, U Gem $A A$ 26,277, YY Gem $A J$ 83,618, RX Her AAp Sup 33,91, TX Her AAp Sup 32, 351, AD Her $\overline{P A S P}$ 90,312, HZ Her = Her X-1 ApJ 208,567, 215, L21, v600 Her AAp 51,1, u Her AAp $61,469,66,161$, VZ Hya ApSpSc 35,249, AAp Sup 32,351, HS Hya AAp 42,303 , $x^{2}$ Hya $\overrightarrow{A A p} 44,4 \overline{45}, 67,15$, AR Lac Ts Shemak 44,3, PASP 88,762, CM Lac AAp Sup 32,351, UV Leo AAp Sup 32,351, XY Leo AJ 83,1452, T LMi PASJ 29,289, $\delta$ Lib ApJ 221,608, RR Lyn Ann Tokyo 16,1, SW Lyn BAC 28,120, B Lyr AZh 52,710, Izv Pulkovo 195,98, ApSpSc 41,121, UX Men AAp 48,49, TU Mon AAp 61,469, VV Mon AAp Sup 33, 323, AO Mon

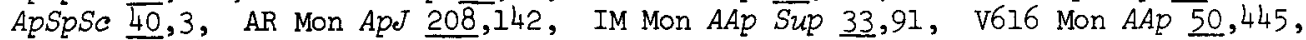
TU Mus $\overline{A A P}$ 45,107, U Oph $\overline{A A p}$ Sup 33,91, WZ Oph AAp Sup 32,351, V1010 Oph ApJ 211,853, VV Ori AAp Sup 33,91, BM Ori ApJ 205,462, AG Peg ApJ 201, 404, AW Peg $\overline{A A p}$ 62,291, X Per AAp 54,817, RT Per ApSpSc 47,277, B Per PASP 87,745, AJ 80,836, $A p J$ 222,L119, фPer ApSpSc 39,495, b Per ApJ 208,152, $\zeta$ Phe AAp 46, 205, SZ Psc AJ 81,250, V Pup AAp 61,275,469, U Sge PASP 88, 688, AAp 61, 469, UU Sge ApJ 223,252, WZ Sge ApJ 219,168, V356 Sgr AAp 61,469, V523 Sco PASP 87,901, v818 Sco = Sco X-I ApJ 207,907, $\mu$ sco AAp 61,469, $\overline{\mathrm{RY}}$ Sct PASP 88,456, RZ Sct PASP 88, 262, CV Ser Af 11,49, CD Tau ApSpSc $\overline{40}, 15, A J$ 81,855, v471 Tau AAp 46, 197, v711 Tau $A J$ 81,771, $\lambda$ Tau AAp 62,291, X Tri Byul2 Abastuman 48,13, TX UMa $A \overline{A p}$ 61, 469, VV uMa $A A p$ Sup 27,285, $\mathrm{CV}$ Vel $A A p$ 44,355, 58,131, BF Vir $A A$ 26,253, DL Vir $A A p$ 61, 107, Z Vul $\overline{A A P}$ 61,469, RS Vul $\overline{A A P}$ 61,469, HD 698 PASP 90,179, HD 47732 ApJ 222,574 , $\mathrm{HD} 7 \overline{7581}=\mathrm{Vel} \mathrm{X}-1 \mathrm{ApJ} \underline{202}, \mathrm{Ll31}, \underline{206}, \mathrm{Ll03}$, Pis AZh 1 Nol2,13, HD 93205 ApJ 207,502, HD 101799 PASP 88,936, HD 159176 BAAS 1,533, HD 160861 AAP Sup 23,277, HD 162724 ApJ 201,792, HD 165052 ApJ 224,558, HD 167771 ApJ 224,558, HD 173198 IBVS 1306, HDE 228766 ApJ 218,431, BD -35357 PASP 89,616, GIiese 815 AJ 83,1445, PG 1413+01 ApJ 224,892, Sk $160=$ SMC X-1 MN 174,29, ApJ 210,I71, 217,186,543, LMC X-4 ApJ $\underline{225}, \overline{548}, 4 \mathrm{U} 1538-52 \mathrm{ApJ} \underline{225}, \mathrm{~L} 63$.

Koch and Koegler (ApJ 2:14,423) illustrated the complicated nature of this system and the great difficulties in its interpretation. For SV Cam, Hilditch reports supporting evidence for a third body with $P \approx 64 \mathrm{y}$, while for the white-dwarf binary V471 Tau, Ollver and Rucinski (IBVS 1444) brought strong arguments against light-time interpretation. A somewhat similar case may be UX UMa, discussed by Quigley and Africano (PASP 90,445); here some doubt was already cast on the triple-system interpretation by Kukarkin ( $M N 180,5 P)$. The system is of great interest, because the period changes are markedly different from those of any other star of this class.

\section{Apsidal Motion (T. Herczeg)}

Several important studies concentrated on the somewhat neglected topic of relativistic periastron advance. A programme of systematic observations of five eccentric systems with relatively long periods, EK Cyg, a CrB, V1143 Cyg, DI Her, and RR Lyn, is being carried out by Koch $(A J$ 82,653). DI Her was also studied by Martynov and 
Khalioullin; they report a marked discrepancy between observed and theoretically predicted rates of relativistic apsidal motion. Koch's value is apparently much closer to theoretical prediction; however, he does not consider the observed magnitude of the effect statistically significant, as yet. V1143 Cyg is also observed by Ebbighausen. Among the cases of "classical" apsidal motion, Y Cyg was discussed by O'Connell (RicA 8,543 ), including results of the Commission campaign, V477 Cyg and DR Vul were re-examined by Todoran $(A A$ 27,59). Apsidal motion was detected for the systems KX Pup and GM Nor (Söderhjelm, $\overline{A A P}$ Sup 22,263) and for V1647 Sgr (Clausen et al., $A A p$ 58,121). Several X-ray binaries were considered (Tanzi and Treves, AAp 60,431), and for $X$ Per, apsidal motion was discussed as possible explanation of the $\overrightarrow{581-d}$ periodicity (Henrichs and van den Heuvel, AAp 54,817). Theoretical apsidal-motion coefficients were calculated by Vila (ApJ 213, 464) for highly evolved objects, such as helium main-sequence and pre-white-dwarf stars.

\section{Proximity Effects and Limb Darkening (M. Kitamura)}

Considerable work was directed towards the theoretical study of tides and related problems. Seguin (APJ 207,848) investigated the effects of tidal distortion on the stability of components against development of turbulence and convection. Horedt (AAp 44,461) studied synchronization of rotation, and Press et al., ApJ 202,I135) circularization of orbits as well, due to tidally induced turbulence. In a comprehensive study, Zahn (AAp 41,329) dealt with the dynamical tide produced by non-adiabatic oscillations of components in the rotating gravitational field. For stars with convective envelopes, Zahn (AAp 57,383) found that the most efficient tidal-friction mechanism is turbulent viscosity retarding the equilibrium tide, while for radiative envelopes the action of radiative damping on the dynamical tide is most efficient. Effects of tidal distortion on velocity curves and ellipsoidal light-variations were considered by Wilson and Sofia (ApJ 203,182) for extreme mass-ratios. Gravity-darkening of W UMa stars was discussed by Ivanov (Perzv 20,99). Eaton (BAAS 9 , 433) noted that not all stars with radiative envelopes obey the von Zeipel law.

Perrenod (ApJ 206,876 ) and Efremov et al. (PerZv 19,407), among others, discussed reflection effects in X-ray binaries. With an application to the bolometric effect in W UMa stars, Pustylnik ( $A A$ 27,25I) studied non-radial energy transport. Kirbiyik and Smith (MN 176,103) modeled circulation currents in irradiated atmospheres; Budding and Ardabili (ApSpSc 59,19) gave a generalized approach to evaluation of the reflection effect.

Lavrov derived linear limb-darkening coefficients for nine systems (ATs 971) and non-linear coefficients for four systems (ATs 990). Discussing the limb-darkening law, Rubashevsky ( $A m A f \frac{27}{9}, 94$ ) found a two-parameter representation as best approximation. Theoretical limb-darkening coefficients were computed by Al-Naimiy (ApSpSc 53,181) for wide ranges of $T, g$, and $\lambda$, and by Manduca et al. (AAp 61,809), who give $\widehat{U B V}$ and $u v b y$ coefficients for late-type giants.

\section{E. Atmospheric Abundances (M. Kitamura)}

Determinations of chemical abundances for double-line binaries were made by several investigators. Heacox $(B A A S 8,522)$ reported ITE analyses for components of the $\mathrm{Hg}-\mathrm{Mn}$ type, including $49 \mathrm{Dra}$ and $6 \overline{6}$ Eri. The system $\delta$ Del, consisting of two nearly identical $\delta$ sct variables, was analysed in detail by Reimers (AAp 53,377), who concluded that both components are hybrias between Am and $\delta$ Sct stars. Kitamura et al. (Ann Tokyo 16,22) found intensity variations for characteristic lines of WW Aur during eclipses, indicating that the distribution of metallic-line features is nonuniform over the surface of either component of this Am binary. $k$ Ari, also with two Am components, was analysed by Mitton (ApA Sup 27,35). Naftilan (ApJ 206,785) found mild metal deficiency for the secondary of U Sge, abundances relative to iron resemble those of Am stars. Contrary to this, Parthasarathy et al. (preprint) report quite normal abundances in $U$ Cep B. More data on abundances in AIgol-type secondaries are 
expected from this Texas group and from Plavec. In AR Lac, Naftilan and Drake (ApJ 216,508 ) found the secondary show high chromospheric activity (strong $H$ and $K$ emission) and be under-abundant in most metals, while the primary has solar-type abundances. Fine-analysis of the F-component of $\varepsilon$ Aur (Castelli, AAp 69,23) gave normal abundances. From spectrophotometry and model-atmosphere calculations, Leushin et al. (Af Issl 9,3) found for the visible component of B Lyr the value 1.55 for the He/H number-ratio. The Copenhagen group derived He abundances for a number of systems, by combining uvby photometry with stellar-model calculations (e.g., Jфrgensen and Gyldenkerne, $A A p$ 44,343). Model atmospheres, illuminated from a companion star, were computed by Muthsam ( $A A \underline{28}, 281)$.

\section{F. Circumstellar Matter (M. Kitamura)}

Observational evidence for circumstellar matter: gaseous disks or rings, streams between components, expanding shells, stellar-wind outflow, etc., continued to increase. In addition to all kind of optical observations, important contributions came from X-ray, satellite UV, and radio data. Thus, for instance, observations with the Copernicus satellite confirmed the existance of a gas stream flowing from Algol B in the direction of A (Cugier and Chen, ApSpSc 52,169), detected mass-flow in UW CMa (McCluskey and Kondo, ApJ 208,760), a stationary H. II-region around V Pup (York et al., ApJ 210,143), and variations in the stellar wind from $\delta$ Ori A (Snow and Hayes, ApJ 226,897). Among optical results we note studies of gas expanding from $\zeta$ Aur and 31 Cyg (Saito and Kawabata, ApSpSe 45.63), evidence for an expanding shell around $\delta$ Ori (Galkina, Izv Krym 54,128), and IR-photometry indicating that a thin shell of graphite grains condensed around the WR binary HD 193793 (Williams et al., MN 185,467).

Wu (ApSpSc 36,407) proposed that double-component emission lines in RW Tau and other binaries primarily originate from gas accumulated at the Lagrangian triangular points. Profiles of lines from disks or rings around one component are computed by Kryz (BAC 30 , in press), using a refined theory. Nariai (PASJ 28,587) studied circulation currents in the common envelope of a contact binary, taking energy transfer into account. Stability against mass overflow in contact binaries was discussed by Nariai and Sugimoto (PASJ $\underline{28}, 593)$; they showed that a binary filling the outer Rochelobe should be unstable against mass loss through $L_{2}$. Particle motions from $I_{2}$ were calculated by Nariai (PASJ 29,263). The interaction with the environment of gas outflowing from young W UMa-stars was studied by Van't Veer (Bamberg Ver 121, 388). Particle mechanics was used by Angeletti (ApSpSe 44,23) and by Piotrowski and Ziolkowski ( $A A$ 28, 295) to elucidate gas flow in semi-detached binaries. The vertical structure of the flow was studied hydrodynamically by Lubow and Shu (ApJ 207,L53). Opaque rings or disks, pictured to surround one component of unusual binaries, such as $B$ Lyr, were approximated by wilson $(A J)$ 80,719) with a torus having elliptical meridian-sections. This geometry for the eclipsing body was used with success to explain the light curve of $\mathrm{V} 356 \mathrm{Sgr}$ (Wilson and Caldwell, ApJ 221,917). Apsidal motion of rings was treated by Castle (PASP $\underline{89}, 862$ ).

The properties of accretion disks, formed around components with small radii, were discussed in a large number of studies, only some of which can be mentioned. Warner (Obs 96,49) reviewed relevant observations. Theoretical studies of gas flow leading to formation of disks were made by Flannery (ApJ 201,66I), Lin and Pringle (IAU Symp 13,237), Shu (IAU Symp 73,253). Models were discussed by Paczynski (ApJ 216 $, 822, A \bar{A} \underline{28}, 91,241)$, Paczynski and Jaroszynski $(A A \underline{28}, 111)$, and Tylenda ( $A A$ 27,235); theoretical UBV colours were computed by Schwarzenberg-Czerny and Rozyczka $(A A \underline{27}, 429)$. Among papers treating structure and stability of disks, we mention lin (Obs 98,208), Livio and Shaviv (AAp 55,95), Paczynski (AA 28,253), Papaloizou and Pringle (MN 181, 441), Piran (ApJ 221,652), Pringle (MN 177,65), Kato (MN 185, 629), Shakura et al. (AAp 62,179), Shulikowskij'(Pis AZh 4,134), Stewart (AAp 42,95, 49,39), Stoeger (MV 182,647), Weber $(B A A S$ 9,632). Accretion onto stars was discussed by Inoue (PASJ 28,293), Kippenhahn and Thomas (AAp 63,265), Neo et al. (PASJ 29,249), Nomoto et al. (preprint), Ulrich and Burger (ApJ 206,509). Of the many papers dealing with 
disk accretion onto magnetic neutron stars and black holes, we quote a few: Aarons and Lea (ApJ 207,914), Bisnovatyi-Kogan and Blinnikov (AAp 59,111), Ghosh et al. (ApJ 217, 578), Lamb et aI. (ApJ 224,969, 225,582), Liang (ApJ 218, 243), Mytrophanov and Tsygan (AAP 70,133), Petterson (ApJ $\underline{214}, 550, \underline{216}, 827, \underline{226}, 253$ ), Shakura and Sunyaev (MN 175, 613).

\section{Structure and Models of Close Binaries}

\section{A. Early-Type Systems}

The structure of the extended atmospheres of Wolf-Rayet stars was studied using eclipses of especially V444 Cyg (Khaliullin and Cherepashchuk, Af 11,593; Limber and Corbin, BAAS 2,305; Hartmann, ApJ 221,193). Hartmann found that the stellar wind from the WN5 component expands at a nearly constant velocity deep in the atmosphere, in contrast to theories of 0-star winds driven by radiation pressure in the lines. An extensive set of $\mathrm{UV}$ observations, including three $\mathrm{WC}+\mathrm{O}$ binaries, were obtained with the TD-1 satellite and analysed in terms of model atmospheres (Willis and Wilson, $M N 182,559)$. Ultraviolet variability was discussed by Burton et al. ( $M N 183,605)$. Spectrophotometry of $\gamma^{2}$ Vel by Moffat (AAP 57,151) revealed variations with timescales 13 - 19 d of certain emission features. Schild and Liller (ApJ 199,432) found no eclipses in CV Ser. Cherepashchuk (Pis $A Z h \underline{2}, 356$ ) and Prilutskii and Usov ( $A Z h$ $\underline{53}, 6)$ drew attention to WR-binaries as potential X-ray sources.

Among the 0-type binaries, especially UW CMa has been the subject of several studies (Hutchings, PASP 89,668; Eaton, ApJ 220,582; Leung and Schneider, ApJ 222,924; Parthasarathy, $M N 185,485)$. Eaton, using $O A O$ light-curves, found a surprisingly sharp limb for the of primary, while the expanding low-density region above is uniform. All three photometric studies agree in that the primary fills or over-flows its Rochelobe. Leung and Schneider found UW CMa a system in over-contact with mass-ratio $m_{2} / m_{1}$ less than unity, contrary to Hutchings.' spectrographic data; the masses suggested are 46 and $34 M_{\odot}$. For V729 Cyg, Leung and Schneider (ApJ 224,565) found the same degree of over-contact (about $30 \%$ ) and about $60 M_{\odot}$ for the primary mass, making it one of the most massive stars known. Bohannan and Conti (ApJ 204,797) suggest this system is on the way becoming a WR-binary. For AO Cas, a third evolved system, only small over-contact was found and masses 25 and $29 M_{\odot}$ (Schneider and Leung, ApJ $223,202)$. Another system of somewhat later type but thought to be very massive, viz. RY Sct, was discussed preliminarily by Ciatti et al. (Bamberg Ver 121,386). IU Aur, for which Mayer $(B A C \underline{27}, 308)$ found the eclipse depths increasing, was studied by Eaton $(A A \underline{28}, 63)$. The depths of minima continue to increase, and the only realistic explanation is a change of orbital inclination due to perturbations from the third body. Nakamura et al. (preprint) computed an evolutionary sequence for SV Cen. The rate of mass transfer for this contact system is believed to be $4 \times 10^{-4} M_{\odot} / y$, which may be 50 times higher than in $\beta$ Lyr. Numerous studies were made of $B$ Lyr itself, some of which have been mentioned earlier in this Report. Huang and Brown completed their investigations with three papers (ApJ 208,780, 218,461, 222,627). The emission spectrum in satellite UV was interpreted by Hack et al. (ApJ Sup 34,565$)$ and Kondo et al. (ApJ 208,468) in terms of a "super-corona" concentrated towards the invisible component but enveloping the whole system. While the possibility that the secondary component is a black hole must still be seriously considered, Jameson and King (AAp 63,285 ) from radio observations argued strongly in favour of a B-star. B Lyr was believed to be a unique object for its UV spectrum, but Plavec and Koch (IBVS 1482) discovered quite similar spectra in $\mathrm{W}$ Ser and some other early-type systems.

We end with a few notes on various kinds of other early-type objects. Several B Cep stars are confirmed binaries; for a recent review, see Lesh and Aizenman Ann Rev AAp 16,215. For one of these binaries, $\alpha$ Vir, the pulsation has decreased so that it is now almost undetectable (Lomb, $M N$ 185,325). LB 3459 , a foreground star in the LMC field, has been shown (Kilkenny et al. (MN 183,523) to be a shortperiod eclipsing system of two 0-type sub-dwarfs. UU Sge, central star of a faint 
planetary nebula (Bond, PASP 88,192) was confirmed to be an eclipsing binary (Miller et al., IAUC 2974; Tsessevich, IBVS 1320). Méndez and Niemela report velocity variations for the central stars of the planetaries NGC 1360 and 2346, indicating that also these objects are close binaries.

\section{B. Algols and Related Systems (M. Plavec)}

Significant progress was accomplished in accumulation of important observational data on semi-detached binaries. The concept of " $R$ CMa systems" (both components grossly undermassive for their luminosities), although almost certainly quite fallacious, continued to stimulate observers to efforts to eliminate the few remaining members of this spurious group. Okazaki (PASJ 29,289) found that T LMi is an ordinary Algol-system with a normal main-sequence primary. Very important is RZ Sct, studied by Wilcken et al. (PASP 88,262 ); here the primary is a B2 II giant. Most important for confrontation with evolutionary calculations are systems where masses of both components can be determined directly from the radial-velocity curves. Most new results come from Popper, using the Varo image-tube at Lick to observe the D-lines of the secondaries; he will soon have complete orbits for the secondaries in 12 new systems. Peculiar is the secondary of the pre-main-sequence system BM Ori in the Trapezium (Popper and Plavec, ApJ 205,462): it appears to be the first observed star to be greatly flattened by differential rotation. While the velocity curves of the secondaries now become increasingly more accessible, some of the primaries are found to give very poor curves. It appears that a circumstellar shell and/or a stream distort the line profiles more or less seriously. Very distorted are $S$ Cnc, AW Peg, TT Hya, RY Per, moderate cases are RY Gem, AD Her, AU Mon, XX Pup, while TW And, TW Dra, RS Vul probably have good photospheric spectra (after Popper). The eccentric orbit of $A D$ Her reported by Batten and Fletcher (PASP 90,312) may be a test case. On the photometric side, Walter reports indications of gas streams and polar-region hot-spots in RW Ara (AAp Sup 26,227), X Gru (AAp Sup 29,51), TW Dra (AAp Sup 32,57), and $X Z \mathrm{Sgr}$. He also found long-period variations of light curves for $\mathrm{TV}$ Cas and $U$ Cep

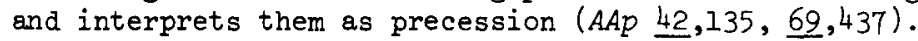

Ultraviolet excesses in U Cep, U Sge, SX Cas, and several RS CVn systems were studied by Rhombs and Fix (ApJ 209,821, 212,446, 216,503) with spectrum scanner. They suggest the excess is due to free-free and bound-free emission associated with the hotter component in the Algols; however, in the RS CVn stars, the excess seems to be associated with the cooler sub-giant. Rucinski observed the spectrum of Algol with the Copernicus sateliite and found that the primary rotates as a solid body and in synchronism with orbital motion. Prilutskij et al. (Pis $A Z h \underline{2}, 294$ ) explained the $\mathrm{X}-\mathrm{ray}$ emission of Algol in terms of an emitting hot spot formed at the surface of the primary by an impinging gas jet.

The idea that at least some Be-stars may be interacting binaries was further followed mainly at Ondrejov. Harmanec et al. announced the binary nature of 4 Her $(B A C 27,47)$, found a period of $87 \mathrm{~d}$ in $88 \mathrm{Her}(B A C \underline{29}, 278)$, and $39 \mathrm{~d}$ in HD 218393. Periodicity and duplicity of 0 And remain controversial (Fracassini et al., ApSpSC 49,145; Harmanec et al., IBVS 1296). Binary nature of the periodic shel1 star HR 2142 wàs confirmed by Peters.

Sub-giant systems with $\mathrm{H}$ and $\mathrm{K}$ emission, more often called RS CVn stars, received broad attention. They appear to evolve from normal main-sequence systems with components of nearly equal mass. Only low rates of mass loss and/or mass transfer are probably required. The many instabilities appear to develop as the components cross the Hertzsprung gap (Popper and Ulrich, ApJ 2l2,LI3l). Weiler (MN 182,77) suggests, from a survey of $\mathrm{H}, \mathrm{K}$, and $\mathrm{H \alpha}$ emissions, an extensive chromospheric activity on the latertype component. Naftilan and Drake (ApJ 216,508) add to it a disk or shell surrounding the secondary in AR Lac. Related to the RS CVn stars are several interesting objects. HD 224085 was shown by Rucinsky (PASP 89,280) to be a strongly spotted, cool but probably young object. CM Dra is the eclipsing binary with the smallest and faintest 
known red-dwarf components; it is probably a population II system (Lacy, ApJ 218,444; Rucinski, $A A \underline{28}, 167)$. Dworetsky et al. (MN 181,13P) discovered a 9-d eclipsing binary, $\mathrm{BD}-3^{\circ} 5357$, containing a red sub-giant and $a$ hot $O B$ sub-dwarf; the evolution of this system poses a problem. Mengel et al. (ApJ 204,488) suggested that in general the sdB stars are produced by mass transfer and mass loss in close binary systems. Great potential significance for our understanding of the helium-rich stars has the discovery (Hesser et al. Nature 262,116; Groote and Hunger, AAp 56,129) that one of the classical objects of this kind, $\sigma$ Ori $\mathrm{E}$, is most likely an interacting binary with ring.

\section{W Ursae Majoris Systems (J.A.J. Whelan)}

Further modifications were made to the basic Lucy (ApJ 151,1123) common convective envelope model of W UMa contact systems. Lucy (ApJ 205, 208), Flannery (ApJ 205,217), Robertson and Eggleton (MN 179,359$)$ discussed a model in which the component stars are not in thermal equilibrium. The system oscillates about a state of marginal contact and spends substantial part of the cycle out of contact with components of very unequal temperatures. Since few, if any such systems are known at the characteristic periods of the models, efforts were made to reduce the out-of-contact part of the cycle. Robertson and Eggleton were able to construct models, with low total angular momentum, which spend only 5\% of the cycle out of contact. The paradoxical possibility arises that as cycling models are constructed which approach $100 \%$ of the cycle in contact, their average properties might formally approach those of the "equilibrium" contact models, which are known to exist only with equal-mass components at the chemical compositions considered. A crucial factor in the cycling-model calculations is the detailed way in which the energy transfer is switched off as the degree of contact is decreased. Not only is some physical picture of energy transfer necessary, but also a very short time-step must be used, since the relative changes of contact depth occur very rapidly and the stellar reaction-times to changing rates of energy and mass flows are very short. The time-step required for accurate computation is probably much shorter than that used so far, and thus the conclusions of Lucy, Flannery, and Robertson and Eggleton must possibly be re-appraised. To cope with short time-steps in a feasible way, Hazlehurst and Refsdal introduced the "response-function method". The stellar response functions (Hazlehurst et al., AAp 58,47) are calculated with the necessary "time resolution" to permit very rapid changes to be followed. Further detailed work is in progress along these lines (Hazlehurst, obs 98,204).

A new, "contact discontinuity" model was introduced by Shu et al. (ApJ 209,536, $214,798, \underline{216}, 517,221,926)$. A discontinuity in the run of physical variables is maintained by fluid flow; the discontinuity provides an extra degree of freedom, which enables models with surface properties in agreement with observations to be constructed. Hazlehurst and Refsdal ( $A A P$ 62, L9) showed that in its first specific form the contact discontinuity would be smoothed out on a very short timescale, thus invalidating Shu et al.'s original model. Shu et al. (ApJ in press) have re-cast somewhat their model; they argue that a contact discontinuity is necessary for the existence of a dynamically stable configuration with unequal-mass components and that fluid flow may maintain a contact discontinuity. Various kinds of observational tests of the theories were collected and discussed by Iucy and Wilson (preprint). The conclusion is that the contact-discontinuity models are in substantial disagreement with observational data, but as several disagreements may no longer apply when the theory has reached its final form, it should not yet be regarded as contradicted.

Several attempts were made to attack the difficult problem of the details of the energy transfer between components of a contact binary (Moses, MN 176,161; Moses and Smith, IAU Symp 13,333; Nariai, PASJ 28,587; Ivanov, Af 12,475; Webbink, ApJ 215,851; Shu et al., ApJ in press). There is not yet agreement about appropriate length-scales, dominant forces, etc. The large-scale circulation model (Webbink), driven by rotation and involving mass motions along the entropy gradients which generate or absorb thermal energy, ties up with the absence of thermal equilibrium in the cycling models. However, Shu et al. argue the mechanism is insufficient. 
The question of which component of a W-type W UMa system is hotter was discussed in detail by Wilson and Biermann ( $A A P$ 48,349). It was concluded, for TX Cnc, that nearly radiative gravity-darkening, with a continuous temperature variation over the components, could account for the light curve as well, or better, than convective gravity-darkening and a hotter secondary. Further work on this subject is in progress by Hilditch (Obs 98,205$)$. Rucinski ( $A A$ 26,227) showed how UV-observations are relevant to the gravity-darkening problem. Woodward and Wilson (ApSpSc 52,387) found that $\mathrm{AK}$ Her is in geometrical contact but with $\Delta T \approx 1000 \mathrm{~K}$ between the components. Wilson (ApJ 224,885) discussed A-type systems selected for having accurately known parameters, and showed that the radii are larger than those of zero-age main-sequence stars.

D. Novae and Dwarf Novae (B. Warner)

The continued increase in observational data on cataclysmic variables, and their similarities to many $X$-ray objects, has generated much interest and attempts at understanding their structure and mechanisms. The three principal reviews on dwarf novae are those by Warner (IAU Symp 73,85), dealing with observations, Bath (IAU Symp 13,173 ), on theories, and Robinson (Ann Rev AAp 14,119). Classical novae were reviewed by Gallagher and Starrfield (Ann Rev $A A p$ 16,171), and two recent semi-popular reviews concern explosions of novae (Bath, QJRAS $\overrightarrow{19}, 442$ ) and the problem of novae versus dwarf novae (Mallama and Trimble, QJRAC 19,430).

Theoretical studies of gas flow between components were quoted in Section 5F; among these, Flannery (ApJ 201,66I) confirmed the empirical hot-spot model. Nelson and $01 s o n$ (ApJ 207,195) reported changes in DQ Her that indicate variations in the rate of mass flow; this was considered further by Nelson (ApJ 209,165). Works on accretion-disk structure were also mentioned in Section 5F; here we add the study of U Gem by Madej and Paczynski (Bamberg Ver 121,313). The structure of dwarf novae at minimum light was elucidated by discussion of multi-colour photometry by Szkody (ApJ $207,824,217,140)$ and refinement of the $U$ Gem model by Smak (AA 26,277). Masses of cataclysmic variables were deduced by Robinson (ApJ 203,485) and Ritter (MN 175,279). Interpretations of the rapid oscillations in DQ Her (Katz, ApJ 200,298; Patterson et al., ApJ 224,570) and in dwarf novae during outburst were assisted by study of $r$-modes (Papaloizou and Pringle, MN 182,423).

Dwarf-nova outbursts and their relationship to binary properties were discussed by Sparks and Starrfield (Liège ColZ 19,407) and Bath (MSAI 45,793). Photometric observations of short and long eruptions of VW Hyi (Haefner et al., AAp in press) provided additional clues. However, there is still no consensus on the nature of the outbursts. Dynamical instabilities of the secondary were further studied by Papaloizou and Bath ( $M N 172,339)$. The most convincing application of the suggestion of dynamical instabilities was made by Webbink (Nature 262,271) to the recurrent nova T CrB.

Much work was performed in studies of classical novae; most of this concerns mechanism of ejection and properties of shells, but there are also results relevant to the binary nature. V1500 Cyg provided a wealth of information. Rapid variations in emission-line profiles indicated rapid changes in the central star (Rush and Thompson, ApJ 21l,184). Rapid flickering and the presence of a $0.14-d$ (or $0.28-d$ ) periodic light-variation (Kemp et al., ApJ 211,L71; Kupo and Leibowitz, AAp 26,181; Young et al., PASP 89,37; Patterson, ApJ 225,954) provided clues to binary nature. Interpretation of infrared and other observations (Ennis et al., ApJ 214,478; Boyarchuk et al., $A Z h$ 54,477; Vrba et al., ApJ 211,480) gave an ejected mass of about $7 \times 10^{-5} M_{\odot}$. A search-light model was proposed by Hutchings and McCall (ApJ 217,775) and further elaborated by Hutchings et al. (ApJ 224,899). Models for ejection from novae (Bath and Shaviv, MN 175,305; Friedjung, MSAI 45,757; Gallagher and Ney, ApJ 204,L35; Clayton and Wickramasinghe, ApSpSc 42,463) were deduced partly from infrared observations; continouos-ejection models are in general favoured. This type of model was also proposed for the slow nova RR Tel by Thackeray (MRAS 83,1). 
Finally we note that the discovery of magnetic close binaries, AM Her, AN UMa, and VV Pup (Section 4C), has closed the gap between conventional cataclysmic variables and $\mathrm{X}$-ray binaries of the Sco $\mathrm{X}-1$ type. In addition to references given already in Section 4C, we mention Chanmugam and Wagner (ApJ 222,641), Cowley and Crampton (ApJ 212,L121), Fabian et al. (MN 179,9P), Greenstein et al. (ApJ 218,L121), Liebert et al. (ApJ 225,201), and Stockman (ApJ 218, I57). The properties of the AM Her variables were recently used by Papaloizou and Pringle (AAP 70,L65) for explaining the different "hump"-periods of VW Hyi.

$\underline{X-r a y ~ B i n a r i e s}$ (E.P.J. van den Heuvel)

For recent reviews of the field, see (1) Proceedings 8th Texas Conference on Relativistic Astrophysics (ed. G. Papagiannis), 1977, Ann. New York Acad. Sci. 302 (especially the papers by Lamb, Rappaport and Joss, Cowley, Flannery, Schreier, Gursky, Ziolkowski, Sanford, Giacconi, Lewin, Ostriker, and van den Heuvel; each paper contains an extensive list of up-to-date references), (2) Physics of Neutron Stars and Black Holes, Proc. 46th Enrico Fermi School of Physics (eds. R. Giacconi and R. Ruffini), North Holland Publ. Co., Amsterdam, 1978. In this Report we only summarize the highlights of the last three years.

Massive $X$-ray Binaries. The most important discoveries concerning the massive X-ray binaries were probably those enumerated and described below.

1. A large number of slow X-ray pulsators with periods in the order of several minutes were recognized. These appear to be more common than the short-period pulsators, such as Her X-1 (1.24 s), Cen X-3 (4.84 s), and SMC X-1 $(0.71 \mathrm{~s})$. Only two more short-period pulsators were discovered: $3 \mathrm{U} 1626-67(P=7.7 \mathrm{~s})$, probably in a lowmass close binary (Joss et al., ApJ 221,645), and $4 \mathrm{U} 0115+63(P=3.6 \mathrm{~s}$ ), in an eccentric massive system ( $P_{\text {orb }}=24.3$ d; Rappaport et al., ApJ 224, II). Presently II slow pulsators are known, most of them associated with massive early-type companions, . either super-giants (e.g. $4 \mathrm{U} 0900-40, P=383 \mathrm{~s}, P_{\text {orb }}=8.96$ d) or B-type emission stars $(3 U 0352+30=X$ Per, $P=835 \mathrm{~s})$. In one case, $3 U 1728-24(P=138 \mathrm{~s})$, the companion seems to be an M-giant (Davidsen et al., Apj $\underline{21}, 866$ ).

2. An important characteristic of all X-ray pulsators is that they spin up rapidly; the timescales are in some cases (3U 1728-24) as short as decades, in others (Cen X-3, SMC X-1) of the order of $10^{3}$ to $10^{4} \mathrm{y}$, in Her $\mathrm{X}-1$ about $3 \times 10^{5} \mathrm{y}$ ( see papers by Schreier and Rappaport and Joss in ref. 1). These large spin-up rates are a clear manifestation of the accretion process, which supplies the compact star with mass and angular momentum. The combination of X-ray luminosity (yielding accretion rate) with spin-up rate (proportional to the mass-transfer rate divided by stellar moment of inertia) provided unambiguous proof that the $\mathrm{X}$-ray pulsators are neutron stars and not white dwarfs (Rappaport and Joss, Nature 266,683).

3. The discovery of the $X$-ray source $O A O 1653-40$ and its positional agreement with $\mathrm{V} 861$ Sco $(P=7.848$ d) provided a second black-hole candidate; the mass function suggests a mass of at least $5 M_{\odot}$ for the compact star (Polidan et al., IAUC 3234). The third black-hole candidate, $\mathrm{Cir} X-1$, was identified with a very faint red star, at least at a distance of $10 \mathrm{kpc}$ (Whelan et al., MN 181,259).

4. The discovery of an orbital period of $1.41 \mathrm{~d}$ in LMC $\mathrm{X}-4$ by Chevalier and Ilovaisky (AAP $59, \mathrm{~L} 9)$ is the first case of a massive binary where binary character was discovered photometrically before it was found in the $X$-rays.

5. The derivation of good optical and X-ray orbits proved to be more complicated than was hoped originally (see Bahcall, Ann Rev AAp 16,241). Much accurate optical photometric and spectroscopic information is required - together with accurate $X-r a y$ data - in order to derive accurate masses of compact stars. So far a complete analysis has been carried out only for the system $4 \mathrm{U} 0900-40=\mathrm{HD} 77581$, by van Paradijs et al. 
(AAp Sup 30,195), yielding a neutron-star mass of $1.74 \pm 0.25 M_{0}$. A second system, where such an analysis seems possible, is SMC X-1 (Hutchings et al., ApJ 217,186); here, however, due to the presence of an extra lightsource in the system near conjunction ( $c f$. van Paradijs and Zuiderwijk, AAp 6I,L19), the errors are considerably larger.

6. The Her $X-1$ system was studied extensively by Boynton and co-workers and by Nelson and Middleditch (see their contributions in ref. 2). In a beautiful analysis Boynton et al. found unambiguous evidence for the presence of a large precessing disk in the system, which causes the $35-\mathrm{d} X$-ray and optical variations.

7. The rather low rate of mass transfer by Roche-lobe overflow in the Her $X-1$ system inspired a detailed study of the effects of hydrodynamics and of angularmomentum transfer on the details of the mass-transfer process. Savonije (AAp 62, 317) showed that especially the effects of hydrodynamics may extend the duration of the phase of very slow mass-transfer $\left(M<10^{-8} M_{0} / y\right)$ by Roche overflow to $\geq 10^{4}$ y in massive systems, provided the massive star is in the phase of hydrogen burning. Therefore, in the short-period massive binaries such as Cen $X-3$ and SMC X-1, Roche-lobe overflow, probably mixed with wind, may be the main source of mass accretion (see also: Conti, $A A p$ 63,225; Ziolkowski, ref. 1).

Low-Mass X-ray Sources, X-ray Bursters, Galactic-Bulge Sources and Globular-Cluster $\mathrm{X}$-ray Sources. After the discovery of the first X-ray burst-source, 3U 1820-30 (in the globular cluster NGC 6624), with the ANS satellite (Grindlay et al., ApJ 205, L127), the SAS-3 team discovered more than 30 new burst-sources. Not more than five of them are in globular clusters. They show a galactic distribution probably characteristic for an old galactic-bulge population (see review by Lewin in ref. 1). At least four bursters have been optically identified, all of them with blue intrinsically faint stars. The spectrum of one of these (4U 1735-44), obtained by McClintock et al. (ApJ $223, L 75)$ is almost identical to that of Sco X-1, suggesting that we are dealing with a low-mass binary system. The time-integrated burst luminosities of all bursters are such that the bursts can be satisfactorily explained by helium-burning flashes on the surface of an accreting neutron star (Joss, ApJ 225, L123). The steady luminosities of the sources, which are about 100 times larger, would, in this model, be due to the accretion process itself. Using all observational evidence, Joss and Rappaport (AAp 11, in press) argue that many of the galactic-bulge sources (including bursters and globular-cluster sources) are short-period binaries, consisting of a low-mass normal star $\left(M \leq 0.5 M_{\odot}\right)$ together with a compact star. The analysis of various capture processes in globular clusters suggests that compact binaries of this type can be formed in sufficient quantities by means of two or three-body captures (Hills and Day, $A p L$ 17,87; Finzi, AAp 62,149; of. Heggie, ComAp 1,43).

\section{Statistical Investigations}

The frequency of binaries and multiples in various stellar samples was studied by several authors mainly by means of new radial velocities. Abt and Levy found among solar-type stars (F3-G2 IV or V) $46 \%$ binaries and 11\% multiples (ApJ Sup 30,273) and for B2-B5 IV or V (including Be) respectively $36 \%$ and $13 \%$ (ApJ Sup 36,241 ). For late $B$-stars (B7-B9) Wolff (ApJ 222,556) obtained a binary frequency of about $45 \%$. In a sample of 18 0-type stars, Bohannan and Garmany (ApJ 223,908) found only two which might be binaries, while several previously considered to be binaries proved to have constant velocities. For OB-stars with enhanced $\mathrm{N}$ or $\mathrm{C}$ lines, Bolton and Rogers (ApJ $222,234)$ estimated the binary frequency to 50-100\%. Binary frequency in IC 4665 was brought down to about $50 \%$ by Crampton et aZ. (ApJ 204,502): previously reported very high frequency is suggested spurious. Among other open clusters searched for binaries we have NGC 6475 (Gieseking, AAp 60,9 ) and NGC 2516 (Gieseking, AAp Sup 32,17). The use of colour-magnitude diagrams of open clusters for obtaining binary frequencies and mass ratios was discussed by Dabrowski and Beardsley (PASP 89,225), Jaschek (AAP 50,185), and by Trimble and Ostriker (AAp 63,433), who especially point to the influ- 
ence of stellar rotation. With reference to globular-cluster X-ray sources, Trimble searched for close binaries in NGC 6809 (BAAS 8,443) and discussed the incidence of close binaries in globular clusters ( $M N 178,335)$. The relation of mass-ratio versus period for eclipsing binaries with $P<5$ d was studied by Sinvhal and Srivastava (ApSpSc 54,239). Trimble (Obs 98,163) discussed the mass-ratio distribution of spectroscopic binaries and found peaks at $M_{2} / M_{1}=0.2$ to 0.3 and 0.9 to 1.0. Van't Veer (AAp 70,91) studied the distribution of mass-ratios for W UMa systems.

\section{Origin and Evolution of Close Binaries}

There are three main theoretical avenues for the formation of binary and multiple stars: (1) collapse and fragmentation of an interstellar cloud into protostars, some of which forming gravitationally bound systems, (2) dynamical evolution of small clusters may as end products have a binary or multiple star, (3) fission may produce very close binaries. Exept perhaps for the very close pairs, the first mechanism is a most promising one, and much work was done in this field (e.g., Larson, IAU Symp 15,255; Mouschovias, ApJ 211,147; Horedt, ApSpSc 54,253; Bodenheimer, ApJ 224, 488; Norman and Wilson, ApJ 224,497). Numerical studies of cluster dynamics have generally assumed that all stars were single at the outset. If binaries already exist in the cluster, much of the $N$-body computations may not be applicable. This is especially so, if a sufficient number of strongly bound pairs exist (Dokuchaev and Ozernoy, $A 2 h$ 55,27; Heggie, Obs 98,206). In globular clusters such pairs are likely to Iorm by tidal captures, and they may also be present primordially. Fabian et al. (MN 172,15P) and Sutantyo ( $A A P$ 44,227) suggested that cluster $\mathrm{X}$-ray sources are close binaries formed from tidal capture of compact objects by ordinary stars. This mechanism was worked out in detail by Press and Teukolsky (ApJ 213,183). Work on the modern versions of the fission theory (Lebovitz, Liège Colz 19,47) has been Iimited. Gingold and Monaghan ( $M N 184,481$ ) studied fission of damped, differentially-rotating polytrops, and Lucy ( $A J \overline{82}, 1013)$ made a successful numerical demonstration of the fission hypothesis, applied to optically thick protostars.

The consequences of mass transfer on the evolution of close binaries were reviewed by Thomas (Ann Rev AAP 15,127), to which we refer for work mainly prior to 1977. Evolutionary sequences for massive binaries with conservative mass-exchange were pursued further by De Grève and de Loore. They found (ApSpSC 50,75) that the sequences bifurcate at an initial primary mass of about $14 M_{\odot}$ : larger masses are progenitors to neutron stars, lower masses end as white dwarfs. In a comparison with observations (De Grève et al., ApSpSc 53,105), it is concluded that conservative mass-exchange, with low initial mass-ratio $\left(q_{i}<0.5\right)$, leads to acceptable first-order models of $W-R$ and massive $\mathrm{X}$-ray binaries. Evolutionary sequences, taking into account mass loss by stellar winds, were calculated by Vanbeveren et al. (AAp in press) for initial masses $30+50,40+20$, and $60+30 M_{\odot}$. The restrictive condition $q_{i}<0.5$ for the $W-R$ systems disappears in these more refined computations. De Grève (preprint) studied similarly a still more massive system, $100+60 M_{\odot}$, which after lobe overflow turns into a helium star with a massive 0-type companion.

Very serious for the theory of Algol systems were the papers by Ulrich and Burger (ApJ 206,509), Flannery and Ulrich (ApJ 212,533), Kippenhahn and Meyer-Hofmeister (AAp 54,539), and Neo et $a$. (PASJ 29,249). All these authors agree that at the phase of rapid mass-transfer, the originally less massive component is uncapable of accomodating the high rate of mass influx, begins to swell and a contact system forms. This is illustrated, for instance, by the model sequence computed by Packet and De Grève (AAp in press) for a $10+3 M_{\odot}$ system, similar to that assumed by Ziolkowski (ApJ $204,512)$ for constructing an evolution model for $\beta$ Lyr. Instead of a system matching B Lyr, Packet and De Grève obtain a contact binary. The evolution subsequent to established contact is less clear, but it is probably rapid mass-loss from the system.

For a low-máss binary $\left(1.50+0.75 M_{\odot}\right.$ ), Webbink (ApJ Sup 32,583) followed evolution from an original detached state to contact. Evolution, when already in contact (Web- 
bink, ApJ 211,881; Rahunen and Vilhu, AAp 56,99) and a possible end-point of contactbinary evolution, namely coalescence (Webbink, ApJ 209,829) were also considered. The possibility of an absence of thermal equilibrium during the contact phase ties up with the cycling models of W UMa systems (Section 6C).

Besides other evolutionary studies, many papers discussed changes of orbital elements due to regular, slow evolution or to catastrophic mass-loss from symmetric or asymmetric supernova explosions. Lack of space prohibits us to enter these fields.

\section{Report of the Coordinated Programs Committee}

(K. Gyldenkerne)

At the Grenoble IAU Meeting it was recommended to carry out coordinated observing campaigns for RX Cas and VV Cep. For RX Cas, the coordinator, D.Ya. Martynov, reports successful UBV-photometry in the USSR: by Zaitseva at the Pulkovo Crimean Station, by Kalv in Tallinn, and by Kumsiashvili at Abastumani; nothing has been reported concerning spectroscopy. From the photometric series, confirmation was obtained for the lengthening of the period. For VV Cep, A. Galatola acted as coordinator and got photometric contributions from observers in USA and Japan, but probably the system has also been observed elsewhere. Wright, Faraggiana and others obtained spectrographic observations; Faraggiana and observers in USA also made IUE spectroscopy. It is suggested to have a discussion on the VV Cep problems at the Montreal Meeting.

Concerning $X$-ray binaries, $Y$. Kondo reports on three coordinated campaigns in 1977. The systems observed and the coordinators are: HDE 226868 (V1357 Cyg) $=\mathrm{Cyg} \mathrm{X}-1$ ( $T$. Bolton), HZ Her $=\operatorname{Her} X-1$ ( $J$. Nelson), and X Per $=4 U 0352+30$ (C. de Loore). About a dozen groups or individual observers participated in each campaign. The X-ray observations of Cyg $X-1$ and Her $X-1$ were performed with SAS-3; the X Per ground-based observations were timed simultaneous with osO-8 $\mathrm{X}$-ray observations.

\section{$\frac{\text { 10. Report of the Committee for Extra-Terrestrial Observations }}{\text { (Y. Kondo })}$}

The Committee continued its usual task of monitoring space experiments that are of interest to the Commission members. In coordination with the Coordinated Programs Committee and IAU Commission 44, we have been involved in various campaigns to observe $X$-ray binaries; a summary of these activities is given in section 9 . For information on satellites that have been active for $X$-ray observations of close binaries and for some observational results, reference is made to section 4D. We add here that the satellite HEAO-B was successfully launched on 1978 November 13. Those interested in observations coordinated with $\mathrm{HEAO} \mathrm{X}$-ray observations may contact $\mathrm{Dr}$. J. Swank at Code 661, Goddard Space Flt. Ctr., Greenbelt, MD 20771, USA.

During the three-year period since 1976, the following two satellites were used to observe close binaries in the ultraviolet (launch dates and organizations/experimenters are indicated):

Copernicus 1972 Princeton University

IUE 1978 NASA (Goddard Space Flt. Ctr.), ESA, British Science Res. Council. In addition, a balloon-borne ultraviolet stellar spectrometer (BUSS) was flown four times to obtain spectra in the mid-ultraviolet. Among binaries observed are $\beta$ Lyr, $\varepsilon$ Aur, $U \mathrm{Sgr}$, and $\mu \mathrm{Sgr}$ (de Jager, Kondo and co-workers).

A larce number of close binaries are being investigated with the two satellites. Copernicus was effective in observing at high resolutions $(0.2$ and $0.05 \AA)$ in the spectral region 1000 - $1400 \AA$. IUE has the capability to observe at slightly lower resolutions ( 0.1 to $0.2 \AA$ in the far-ultraviolet and 0.2 to $0.3 \AA$ in the mid-ultraviolet), alternatively, it can observe much fainter objects at a resolution of 6 to $7 \AA$ in the range $1200-3100 \AA$. 
The results already published from Copernicus data include papers on $\beta$ Lyr, B Per, UW CMa, and HD 153919 (4U 1700-37). R. Polidan prepared the following list of close binaries observed with Copernicus since 1976 January 1 : $\lambda$ And, UX Ari, 54 Cam, $R$ CMa, AO Ces, AR Lac, HK Lac, 17 Lep, $\delta$ Lib, $\theta$ Mus, X Per, $B$ Per, V Pup, $\mu \mathrm{Sgr}, \mathrm{U} \mathrm{Sgr}, \mathrm{V} 861 \mathrm{Sco}, \mathrm{V} 711 \mathrm{Tau}, \lambda \mathrm{Tau}, \varepsilon \mathrm{UMi}, \gamma^{2}$ Vel, HR 2142, 4665, 5110, 7275, 8703, HD 72754, 77581, 153919; and a list of observed, possibly interacting binaries: $\zeta$ And, $\pi$ Aqr, $\alpha$ Aur, $\alpha$ CMa, $\tau$ CMa, $u$ Cyg, 66 Oph, $\delta$ Ori, $n$ Ori, $\theta$ Ori, 1 Ori, $\lambda$ Ori, $\pi^{5}$ Ori, $\sigma$ Ori, $\epsilon$ Per, o Per, $\psi$ Per, $\delta$ Pic, $\alpha$ Sco, $\beta^{1}$ Sco, $\mu$ Sco, $\pi$ Sco, $\alpha$ Vir, HR 4830, 7084, HD 50896. B. Perry compiled the following list of examples of mostly non-X-ray eclipsing binaries observed with the IUE by guest observers, Hack: $E$ Aur, VV Cep, CH Cyg, B Lyr, U Sgr, HD 192909; Koch: RX Cas, RW Cas, SX Cas, V382 Cyg, V1073 Cyg, TT Hya, AR Pav, RW Per, V Sgr, U Sgr, V453 Sco; Kondo: UW CMa, AO Cas, U Cep, 31 Cyg, 32 Cyg, 8 Lyr, $\theta$ Mus, $n$ Ori, $\delta$ Pic, $\mu$ Sgr, u Sgr, V861 Sco, $\gamma$ Vel, HD 153919; Plavec: RX Cas, W Cru, V367 Cyg, 17 Lep, $x$ Oph, AG Peg, RW Per, 3 Pup, $\mu \mathrm{Sgr}, \mathrm{V} 356 \mathrm{Sgr}, \mathrm{RZ}$ Sct, W Ser, HD 161114, 163296, 206773. Additional interacting binaries, such as SS Cyg, were also observed with the IUE in other guest-observer programs but are not included here, X-ray emitting binaries observed with the IUE in a collaborative program between USA and Europe are given as follows (list prepared by R. Davis): good phase-coverage for the systems HZ Her, V1357 Cyg, V818 Sco, HD 77581, HD 153919, and varying degrees of phase-coverage for SS Cyg, AM Her, X Per, Sk 160, HD 152667, LMC X-4.

Highlights of $\mathrm{X}$-ray binary observations obtained during the initial high-priority period of the IUE are presented in an article by Dupree et al. in a special issue of Nature ( 1978 October 5). Among preliminary reports is Hack's detection of the hot companion in $\varepsilon$ Aur. Plavec reports detection of strong emission lines of hot plasma in $\mathrm{W}$ Ser, $\mathrm{V} 367 \mathrm{Cyg}$, and $\mathrm{W}$ Cru. Most prominent lines in the region $1200-2000 \AA$ are due to $\mathrm{NV}$, Si IV, C IV, Si III, AI III, and He II. On the whole the spectra are similar to that of $\beta$ Lyr. Koch and Sobieski detected similar spectra in RX Cas and SX Cas. Thus, it is established that a whole class of interacting binaries shows this type of spectrum. Plavec speculates on several possible explanations, including the presence of black holes. Kondo and McCluskey observed spectral profiles indicating mass flow in a number of cases, including $U$ Sgr, $\mu \mathrm{Sgr}$, V86I Sco, B Lyr, UW CMa, and U Cep. Those interested in learning more about these satellites' observations may contact Dr. R. Polidan (Copernicus), Code 410, and Dr. R. Perry (IUE), Code 685, both at Goddard Space Flt. Ctr., Greenbelt, MD 20771, USA.

\section{$\frac{\text { 11. Report of the Working Group on RS CVn Binaries }}{\text { (D.S. Hall) }}$}

This Working Group was conceived at the end of the Grenoble Meeting to stimulate interest in $\mathrm{RS} \mathrm{CVn}$ systems, make it known which astronomers are involved, define some of the important problems, minimize unnecessary duplication, and help with cooperative observing programs. To date 11 Circulars have been distributed to about 35 members in the Western Hemisphere and (with the help of $\mathrm{M}$. Rodono) to about 15 in the Eastern Hemisphere. Members are encouraged to announce research-in-progress and look for published results in the Bibliography and Program Notes on Close Binaries. We still need one light curve per year outside eclipse for the about 20 most important systems in order to follow the wave migration continuously; with less frequent observations, the phasing.can become ambiguous. The "First RS CVn Workshop" was held 1978 April 6 and 7 at New Mexico Institute of Mining and Technology, Socorro, NM, with D.S. Hall scientific chairman, D.M. Gibson local chairman, and 27 participants. A summary of the discussions was distributed as Circular No. 9. There we arranged to have a special issue of the Astron. J. (December 1978) devoted to the large 1978 February 20 radio outburst of V71I Tau (HR 1099) and arranged to have a "Second RS CVn Workshop" at the Montreal Meeting, with P. Feldman local chairman. 